\title{
Using Fuzzy Linguistic Representations to Provide Explanatory Semantics for Data Warehouses
}

\author{
Ling Feng, Member, IEEE, and Tharam S. Dillon, Fellow, IEEE
}

\begin{abstract}
A data warehouse integrates large amounts of extracted and summarized data from multiple sources for direct querying and analysis. While it provides decision makers with easy access to such historical and aggregate data, the real meaning of the data has been ignored. For example, "whether a total sales amount 1,000 items indicates a good or bad sales performance" is still unclear. From the decision makers' point of view, the semantics rather than raw numbers which convey the meaning of the data is very important. In this paper, we explore the use of fuzzy technology to provide this semantics for the summarizations and aggregates developed in data warehousing systems. A three layered data warehouse semantic model, consisting of quantitative (numerical) summarization, qualitative (categorical) summarization, and quantifier summarization, is proposed for capturing and explicating the semantics of warehoused data. Based on the model, several algebraic operators are defined. We also extend the SQL language to allow for flexible queries against such enhanced data warehouses.
\end{abstract}

Index Terms—Data warehouse, semantic model, algebraic operator, extended SQL, fuzzy set, membership function.

\section{INTRODUCTION}

$\mathrm{D}$ ATA warehouses have gained in importance in recent years [53], [9], [41]. A data warehouse integrates large amounts of enterprise data from multiple and independent data sources consisting of operational databases into a common repository for querying and analysis. Often, data warehouses are designed for online analytical processing, where the queries aggregate large volumes of data in order to detect trends and anomalies. To reduce the cost of executing aggregate queries in such an environment, warehousing systems usually precompute frequently used aggregates and store each materialized aggregate view in a multidimensional data cube. These data cubes group the base data along various dimensions, corresponding to different sets of group-by attributes, and compute different aggregate functions on measures. Sum, avg, min, max, and count are the most commonly used aggregate functions. For example, consider a data warehouse with historical sales data for a large chain of department stores. Assume that all the member stores sell the same products no matter where (in the east, west, north, or south) they are located. Sales data is collected daily. At the end of each year, each member store will report the total sales amount of each product to the headquarters. Fig. 1 shows part of a multidimensional aggregate view, where the measure Sales is functionally determined by three-dimensional attributes Product, Year, and Store. Detailed summary data is given in Table 1.

- L. Feng is with the Department of Computer Science, University of Twente, Netherlands. E-mail: ling@kub.nl.

- T.S. Dillon is with the Deparment of Computer Science and Computer Engineering, LaTrobe University, Bundoora 3086, Australia.

E-mail: tharam@cs.latrobe.edu.au.

Manuscript received 10 Dec. 1999; revised 12 Oct. 2000; accepted 28 Mar. 2001.

For information on obtaining reprints of this article, please send e-mail to: tkde@computer.org, and reference IEEECS Log Number 111070.
Executives at the headquarters can then use this data to analyze the activities of the chain stores.

Example 1. One indicator of the activities of the chain stores is the sales performance, which can be obtained via the following query:

$Q_{1}$ : "For each member store, find the total sales amount in 1998."

Also, users can query those products with total sales above a certain value:

$Q_{2}$ : "For each member store, find products whose total sales in 1998 were above 230."

The growth trend of products in sales can be obtained by queries such as:

$Q_{3}$ : "Find products whose sales difference in the past two years was within 100."

As data warehousing systems provide easy access to both historical and aggregate data which are derived across several data sources beforehand, the analysis-oriented queries such as those given in Example 1 can often be evaluated much more cheaply using warehouse data than using the base relations.

Nowadays, data warehousing has become a key technology to assist management in making quick and competitive business decisions. From the viewpoint of both industry and the research community, data warehousing related problems have sparked vigorous discussions [54], [53], including the use of multidimensional models and OLAP [1], [39], [24], [50], materialized view selection [27], [31], [62]. [28], warehouse maintenance [25], [26], [70], [32], [33], [41], [46], [63], [69], [21], query language and processing [11], [7], [8], [23], [30], [29], [20], [48], [67], [68], [13], and physical warehouse design [55], [34], [46], [44], [38]. With more and more data warehousing tools and products developed and applied to decision support systems, we 


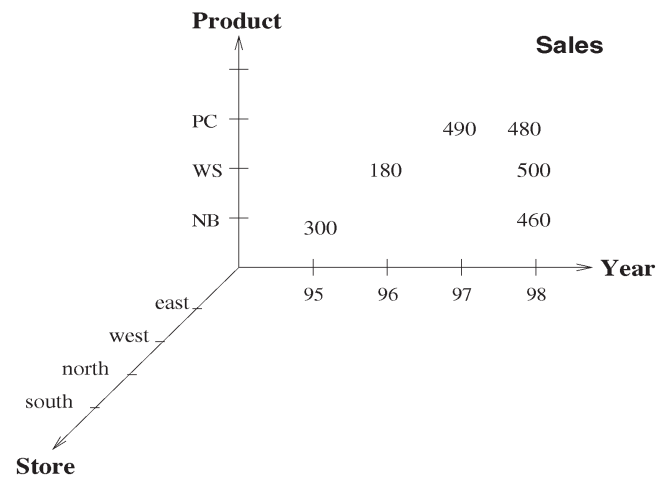

Fig. 1. A three-dimensional aggregate view for a large chain store.

ask one question: Is it enough? or, What technologies do we still need for data warehousing?

\subsection{Inadequate Semantics in Data Warehouses}

Currently, most analysis that data warehousing systems perform is numerically oriented such as counting the total sales of products within a certain period, calculating the average sales of products in a certain area, etc. Although the numbers give some indications of the sales behavior, their real meaning is missing. For example, whether a total sales 1,000 items indicates a good or bad sales performance is still unclear. From a manager's viewpoint, the semantics conveyed by linguistic labels are actually more natural, meaningful, and understandable. In fact, much of human reasoning in real life involves the use of linguistic words rather than rigid numbers [10]. The meaning of these linguistic words will necessarily involve an agreed interpretation between users and knowledge workers which is captured by agreement over the membership functions.

Example 2. In addition to querying detailed sales amounts via $Q_{1}, Q_{2}, Q_{3}$ in Example 1, users would also like to know the sales performance expressed using natural language terms like:

$Q_{1}^{\prime}$ : "How was the sales performance $(\mathrm{good} / \mathrm{bad} / \mathrm{med}$ ium) of each member store in 1998?"

$Q_{2}^{\prime}$ : "For each member store, find products whose sales performance in 1998 was good."

$Q_{3}^{\prime}$ : "Find the products whose sales in the past two years were nearly unchanged (accepting minor difference)."

To answer the above queries, apparently, warehousing systems need more techniques to build up the meaning of numbers so as to reflect the users' perception of real-world data and establish a closer human-computer interaction consequently. This has been constantly missing before. It is worth pointing out that in the database area, the term semantics is frequently taken to mean the metadata that gives structural semantics to the stored data. We use it here to provide explanation for the meaning of data in the data warehouse, i.e., explanatory semantics.

Fuzzy technology provides a framework for modeling the interface between human conceptual categories and data. As Zadeh says [65], "It reduces cognitive dissonance in problem modeling so that the way that humans think about the decision process is much closer to the way it is represented in the
TABLE 1

Summary Data of the Chain Store

\begin{tabular}{|c|c|c|c|}
\hline \hline \multicolumn{3}{|c|}{ Dimensions } & Measure \\
\hline Product & Year & Store & Sales \\
\hline PC & 1997 & east & 490 \\
PC & 1997 & west & 200 \\
PC & 1998 & south & 480 \\
PC & 1998 & west & 450 \\
PC & 1998 & east & 300 \\
PC & 1998 & north & 50 \\
Workstation & 1998 & east & 500 \\
Notebook & 1998 & east & 460 \\
.. &.. & .. &.. \\
\hline \hline
\end{tabular}

machine." Since its introduction by Zadeh [64], fuzzy technology has been widely used in a number of areas [10]. In the fuzzy database community, research on fuzzy databases has been conducted for about 20 years, with the emphasis on handling of imprecise, uncertain or fuzzy data, flexible querying, and defining and using fuzzy dependencies [40], [16], [12], [6], [4], [5]. However, compared with the areas of expert and control systems, fuzzy database systems have not found wide applications. Indeed they have remained outside the main stream database world. This might be due to the lower expressed demand for uncertain and imprecise representation and management in the database field. Usually, a simple NULL value is adopted in databases to describe these unknown or uncertain data [49], [45]. In contrast, although a data warehouse is an integration of a number of databases, it aims at providing online analysis and direct querying to business managers who would prefer more general linguistic labels as well as crisp numbers. Moreover, applying fuzzy technology to capture the semantics of warehoused data can aid users to easily formulate retrieval criteria and make meaningful decisions. In operating a decision support system, a human's thinking tends to be nonprecise and usually cannot easily decide query conditions with crisp values, especially when these conditions come from his or her very complex image. For example, with a sales support system which is used to hit high potential customers, the sales person must think about how to get good customers when selling high-grade products. In such an uncertain environment, an intuitive interface to make a query condition by using linguistic labels such as "good," "high," etc. is more convenient for human beings [42]. Besides, the matching grades between a query condition and the responses, returned from a fuzzy retrieval, enable one to rank-order the $N$ best replies and get target data easily. Taking a bargain sales mailing system as an example [42], suppose a company plans to send 1,000 pieces of mail according to the budget for sales promotions. However, at the beginning, the number of customers retrieved could be 1,200. After changing the query criteria, the number may become 850 according to the new query condition. In order to reach the desired number of answers, users need to tune query criteria and formulate a sequence of queries. In contrast, a fuzzy query can calculate the matching grade of each 
customer with the query condition, rank the results in a descending order of grades, and return the 1,000 top-most customers to assure one gets the high potential customers.

We believe that with the aid of fuzzy techniques, data warehousing systems can offer more flexible and powerful decision support than currently exists.

\subsection{Our Work}

In this paper, we provide an investigation of the potential applications of fuzzy technology in data warehousing systems. A three-layered data warehouse semantic model is proposed, where we structure the warehouse summary data into three levels of abstraction, namely, quantitative (numerical) summarization level, qualitative (categorical) summarization level, and quantifier summarization level. Traditional aggregate views of data warehouses, like summary data in Table 1, constitute the bottom quantitative (numerical) summarization level in this architecture. Further generalization on these numerical summary data in the form of linguistic terms forms the middle qualitative (categorical) summarization level. For example, products can be categorized by good (medium or bad) sales performance at this level. Furthermore, besides the numerical and categorical summarization on each individual record, business managers may also want to have some insight into the overall performance of a group of records using statements like "Most (Half or Few) of products' sales behavior is good (medium or bad)." Hence, the third level provides quantifier summaries about the databases in quantifier terms that are easy and natural for people to comprehend.

To facilitate the construction of the above three summarization levels, two operators FUZZ-TERM and FUZZ-QUANTIFIER are introduced to enable warehouse users to declare a basic set of linguistic terms and quantifiers that are of interest and importance to the problem solving and decision making process. A set of algebraic operators based on the proposed semantic model is provided for operating the enhanced data warehouses. We also extend the SQL language to allow for flexible queries on different warehouse summarization levels.

The remainder of the paper is organized as follows: In Section 2, we provide a brief review of the basic concepts of fuzzy sets, membership functions, and associated operations relevant to our work. With the aid of fuzzy techniques, a three layered data warehouse semantic model is proposed to provide semantics to the warehoused data in Section 3. We describe a set of algebraic operators and query facilities against such enhanced data warehouses in Section 4 and Section 5 , respectively. Section 6 concludes the paper with a brief discussion of future work.

\section{Brief Review of Basic Fuzzy Set Theory}

In this section, we review basic fuzzy set theory, including membership functions, fuzzy sets, and associated operations which are of particular applicability in the area of data warehouses. More complete introductions to fuzzy set theory can be found in a number of excellent texts [10], [19], [71], [36], [51].

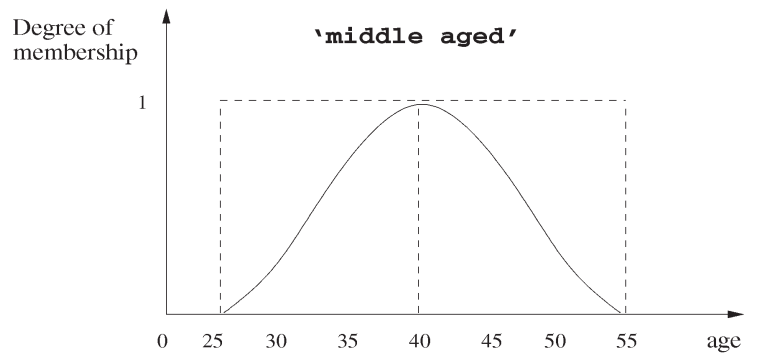

Fig. 2. A bell-shaped membership function for "middle-aged."

\subsection{Fuzzy Sets and Membership Functions}

The concept of a fuzzy set extends the notion of a regular crisp set in order to express classes with ill-defined boundaries, corresponding in particular to linguistic values such as "tall," "young," "well-paid," "important," etc. Within this framework, there is a gradual rather than sharp transition between nonmembership and full membership. A degree of membership is associated with every element $x$ of the universal set $X$. It takes its value in the interval $[0,1]$ instead of the pair $\{0,1\}$. Such a membership assigning function $\left(\mu_{A}: X \rightarrow[0,1]\right)$ is called a membership function and the set defined by it is a fuzzy set [36], [10]. Fig. 2 shows an example concept "middle-aged" modeled as a bell curve.

The concept known as the alpha-level set or " $\alpha$-cut" of a fuzzy set $A$ implies a subset, made of those elements whose membership degree is over or equal to

$$
\alpha:\left(A_{\alpha}=\left\{x \in X \mid \mu_{A}(x) \geq \alpha\right\}\right) .
$$

A fuzzy predicate expresses the degree to which the arguments satisfy the predicate. An elementary predicate (single or multivariable) allows for comparison between variables and constants or between variables [4]. Fig. 3 describes the predicate "price $=$ medium" with $\alpha$-cut 0.2. In this case, any price between 24 and 26 is considered "medium" and fully satisfies the predicate. A price of 20 or 30 satisfies this same predicate at a degree of 0.2 , whereas any price under 19 or over 31 is not "medium" at all.

\subsection{Fuzzy Set Operations}

Fuzzy sets can be combined in an analogous way to ordinary crisp sets by means of set operations. Besides, there are some additional operations based on membership values of a fuzzy set, which have no correspondence in crisp sets.

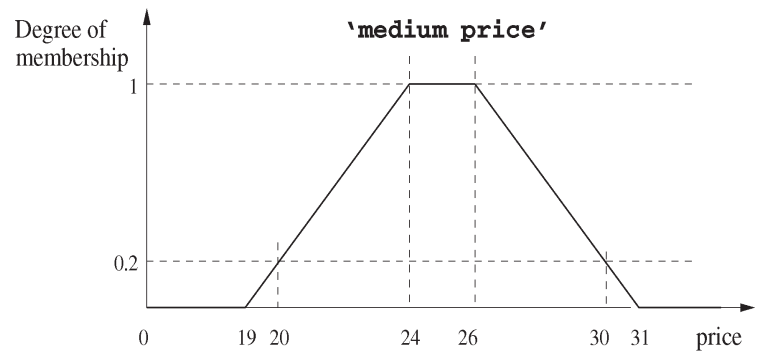

Fig. 3. A trapezoidal-shaped membership function for "medium-price." 


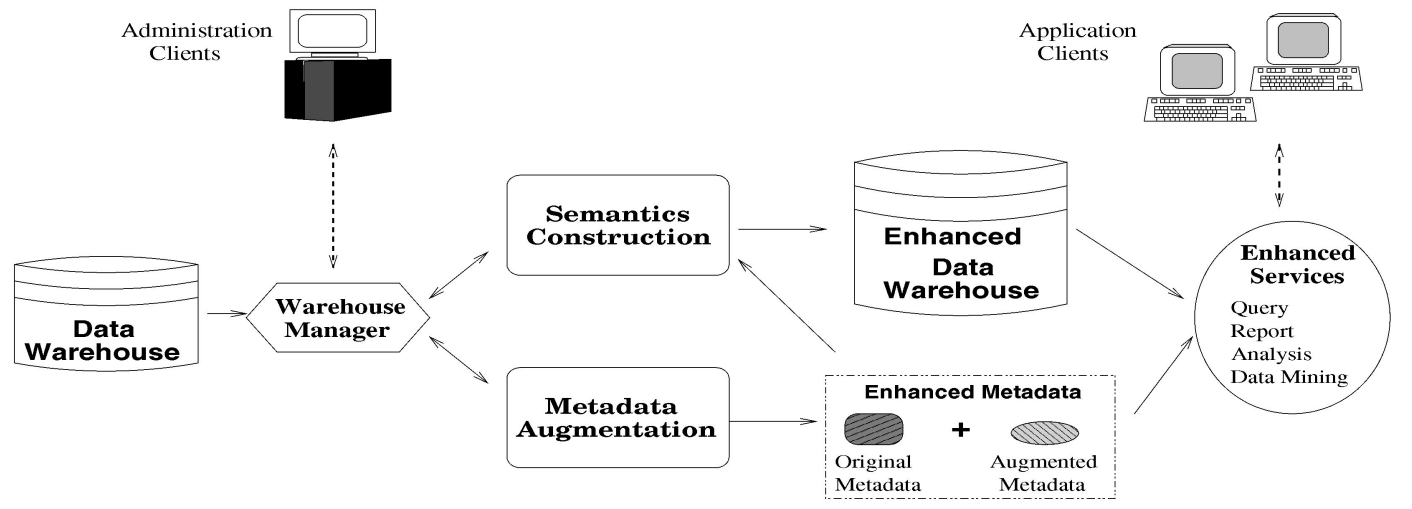

Fig. 4. A framework for enhancing the semantics of a data warehouse.

1. Union and Intersection of Fuzzy Sets. Let $\mu_{A}$ and $\mu_{B}$ represent the membership functions of the fuzzy sets $A$ and $B$, defined over the universe $X$. The classical set union $(\cup)$ and intersection $(\cap)$ can be extended by the following formulas:

$$
\begin{aligned}
& \forall x \in X, \mu_{A \cup B}(x)=o p_{1}\left(\mu_{A}(x), \mu_{B}(x)\right), \\
& \text { where } o p_{1} \text { is a triangular conorm; } \\
& \forall x \in X, \mu_{A \cap B}(x)=o p_{2}\left(\mu_{A}(x), \mu_{B}(x)\right), \\
& \text { where } o p_{2} \text { is a triangular norm. }
\end{aligned}
$$

Among the pairs conorm/norm of operators $\left(o p_{1} / o p_{2}\right)$, the $\max / \mathrm{min}$ proposed by Zadeh [64] preserves most of the usual properties of intersection and union.

$$
\begin{aligned}
& \forall x \in X, \mu_{A \cup B}(x)=\max \left(\mu_{A}(x), \mu_{B}(x)\right) \\
& \forall x \in X, \mu_{A \cap B}(x)=\min \left(\mu_{A}(x), \mu_{B}(x)\right) .
\end{aligned}
$$

There are a number of alternative functions proposed to represent fuzzy set union and intersection [19], [56]. For example, in the case of intersection, a product definition $\mu_{A}(x) * \mu_{B}(x)$ can also be considered.

2. Complement of a Fuzzy Set. The complement $\bar{A}$ of $A$ is defined by the following membership function [64]:

$$
\forall x \in X, \mu_{\bar{A}}(x)=1-\mu_{A}(x) .
$$

For ordinary crisp sets $\bar{A} \cap A=\phi$; however, this is not generally true for a fuzzy set and its complement. Since fuzzy sets have imprecise boundaries, we cannot place an element exclusively in a set or its complement.

3. Difference of Fuzzy Sets. Since $A-B=A \cap \bar{B}$, the difference of two fuzzy sets delivers a fuzzy set where

$$
\forall x \in X, \mu_{A \cap \bar{B}}(x)=\min \left(\mu_{A}(x), 1-\mu_{B}(x)\right) .
$$

4. Concentration and Dilation of a Fuzzy Set. The concentration operation concentrates fuzzy elements by reducing the membership grade proportionally more for elements that have smaller membership grades.

$$
\mu_{C O N(A)}(x)=\left(\mu_{A}(x)\right)^{2} .
$$

In contrast, the dilation operation dilates fuzzy elements by increasing the membership grade more for the elements with smaller membership grades.

$$
\mu_{D I L(A)}(x)=\left(\mu_{A}(x)\right)^{1 / 2} .
$$

The CON and DIL operators have no counterparts in ordinary set operations, and are commonly used to represent linguistic hedges that act as modifiers to linguistic variables expressed in fuzzy sets. For example, the CON operator can be used to approximate the effect of the linguistic modifier hedge "very." In other words, $\operatorname{CON}(A)=\operatorname{very}(A)$. Also, $\operatorname{DIL}(A)$ can be used to interpret the linguistic hedges such as "approximate $A$ " or "more-orless $A^{\prime \prime}$ [45].

The concepts of fuzzy sets and associated operations presented here form the basis of our work on the semantics of data warehousing systems.

\section{Providing Explanatory Semantics to Data WAREHOUSES}

In this section, we explore the use of fuzzy technology to enhance the semantics of data warehousing systems. After introducing the framework of our approach, we give a detailed description of a multilayered data warehouse semantic model. A cluster-based strategy is also presented to address the traditional membership setting problem in the proposed data warehouse semantic model.

\subsection{The Framework}

Fig. 4 outlines the major components and their relationships involved in capturing and explicating the semantics for a data warehousing system. In addition to the ordinary duties performed by a warehouse manager, two new functions, Semantics Construction and Metadata Augmentation, are introduced in order to enhance warehouse semantics, and, hence, provide application clients with meaningful business views and effective decision-support services. These components cooperate as follows:

- The Warehouse Manager is responsible for storing, managing, and maintaining data in the warehouse. In situations which require applications of human 


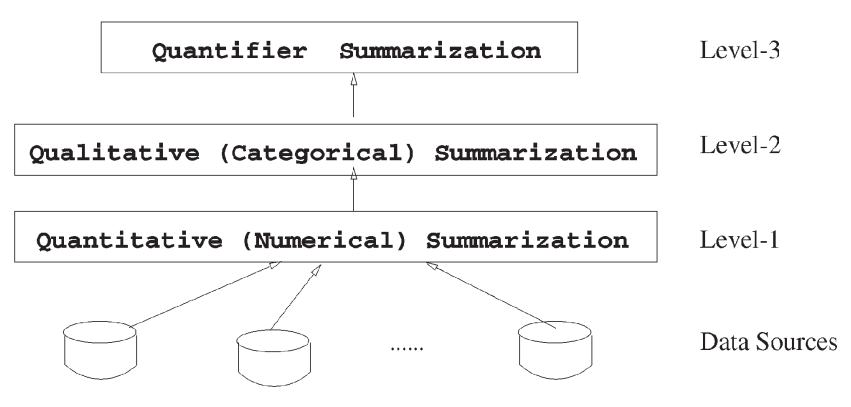

Fig. 5. A three-layered data warehouse summarization architecture.

approximate reasoning and perceptual views, the warehouse administrator will instruct the warehouse manager, which will in turn initiate the semantic building process as follows:

- The Semantics Construction step is to provide different levels of data views and categorizations in terms of natural-language-like labels, like "good," "bad," "most," "almost all," or "few," etc. This involves the fuzzification of relevant data in the original warehouse. The traditional fuzzy set and membership function techniques are applied.

- Metadata is an essential element of the warehousing system. It assists users in identifying, locating, and making full use of warehouse data for their business activities. Besides the traditional administration, business, and operation related metadata [9], [22], the data used to describe and interpret the enhanced data structures and semantics are also recorded and stored. This kind of metadata describes various subjective categories and views of business data (e.g., "good" or "bad" sales performance), together with the applicable membership functions and operations (e.g., the comparison of two products' sales performance-“comparable," "close to," or "greatly different," etc.). The Metadata Argumentation component is responsible for the creation and maintenance of such kind of enhanced metadata.

In the following section, we will elaborate an enhanced semantic model for data warehouses in detail.

\subsection{A Three-Layered Data Warehouse Semantic Model}

In the model, the semantics of warehouse data are explicated at three different summarization levels_quantitative (numerical) summarization level, qualitative (categorical) summarization level, and quantifier summarization level, as shown in Fig. 5. Fig. 6 is a schematic representation of the three summarization levels on a three-dimensional data cube.

At each level, data is organized in one or more multidimensional cubes, corresponding to that level. In general, a cube has three basic components: a set of dimensions, a measure, and a mapping from a set of dimensions to a measure. The dimensions are assumed to uniquely determine the measure. Throughout the paper, we refer to the dimensions as $D_{1}, \ldots, D_{n}$. For each dimension $D_{i}$, we use $\operatorname{dom}\left(D_{i}\right)$ to denote the domain, from which values are taken.

\subsubsection{Level-1: Quantitative (Numerical) Summarization}

This level is made up of various aggregate views in traditional data warehouses. Since the view data is often calculated and derived by numerical aggregate functions like sum, avg, count, min, max over base resources, we name this level as the quantitative (numerical) level.

Definition 1. An n-dimensional quantitative data cube $\mathcal{C}_{q}^{n}$ can be defined as a mapping function $\mathcal{F}_{q}\left(\mathcal{C}_{q}^{n}\right): \operatorname{dom}\left(D_{1}\right) \times$ $\ldots \times \operatorname{dom}\left(D_{n}\right) \rightarrow \mathcal{V}$, which maps a set of dimensional values to a set of scalar values and a NULL value.

$\mathcal{F}_{q}\left(\mathcal{C}_{q}^{n}\right)\left(d_{1}, \ldots, d_{n}\right)=v$ refers to the measure $v \in \mathcal{V}$ at position $\left(d_{1}, \ldots, d_{n}\right)$ of the cube, where $d_{1} \in \operatorname{dom}\left(D_{1}\right)$, $\ldots, d_{n} \in \operatorname{dom}\left(D_{n}\right)$, and $\mathcal{V}$ is a set of scalar values and a NULL value. $v=N U L L$ indicates the measure

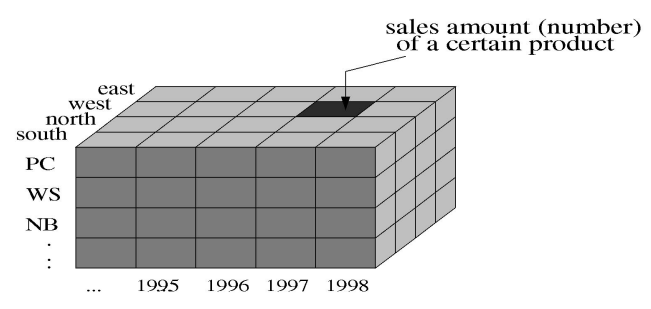

Leve1-1

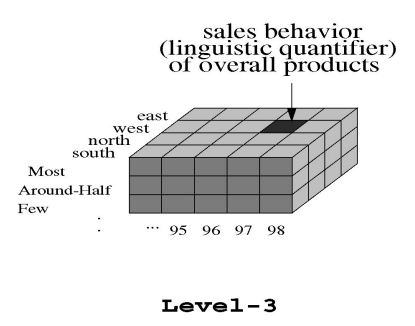

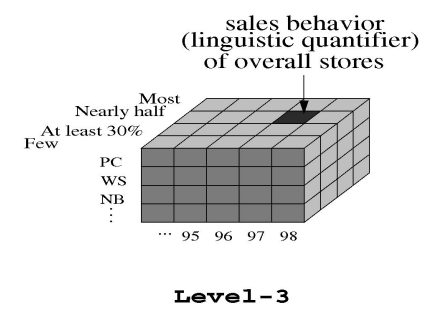

Fig. 6. A schematic representation of different summarization levels in a data warehouse. 

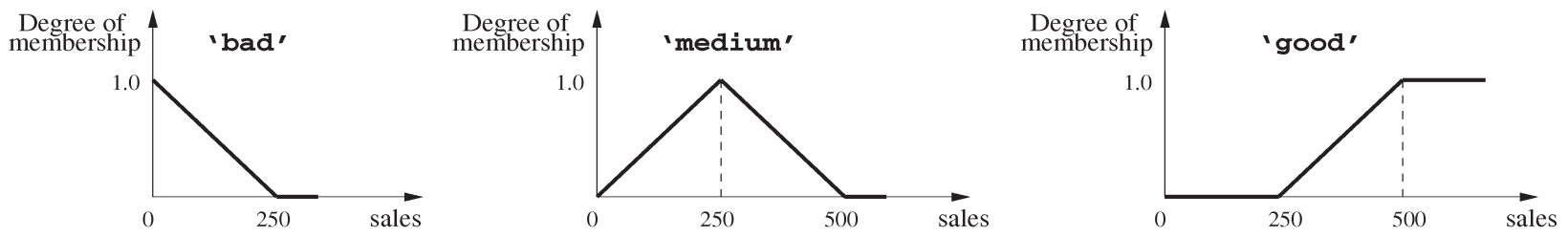

Fig. 7. The membership functions defined in Example 4.

corresponding to a certain combination of dimensional values does not exist in the cube, i.e., indicating an empty measure.

Example 3. Table 1 illustrates a three-dimensional quantitative data cube $\mathcal{C}_{q}^{3}$ stored using a database table, in which

$$
\mathcal{F}_{q}\left(\mathcal{C}_{q}^{3}\right)(P C, 1997, \text { east })=490,
$$

but

$$
\mathcal{F}_{q}\left(\mathcal{C}_{q}^{3}\right)(P C, 2001, \text { east })=N U L L
$$

As the approaches to numerically aggregating data are widely known and published in the literature, we will not discuss this level any further here.

\subsubsection{Level-2: Qualitative (Categorical) Summarization}

Considering the fact that a human's perception and thinking are generally based on linguistic labels instead of crisp numbers, we further summarize the numerical data at Level-1 into descriptive and categorical concepts so that the way decision makers think about a problem's solution is much closer to the way it is represented in data warehouses. Here, we apply the fuzzy set techniques to facilitate this process [64], [65]. For example, according to the total sales of products at Level-1, we can categorize different products by "good" ("medium" or "bad") sales performance at Level-2 using certain membership functions. Later, users can directly invoke these linguistic terms to query the warehouse data.

As a measured attribute can usually be associated with many concepts (e.g., sales can be described by the performance of "very good," "good," "medium," "bad," or "very bad" or it can be expressed through the comparison with its previous behavior like "greatly changed," "slightly changed," or "nearly unchanged,") an operator FUZZ-TERM is introduced to enable the warehouse administrator to indicate a basic set of linguistic terms that are of interest and importance to the decision making process, and they span the universe of discourse for that attribute.

\section{FUZZ-TERM}

$<$ linguistic term set $><$ membership function set $>$

$\mathrm{ON}<$ measurement attribute $>$ FROM $<$ table $>$

Example 4. Consider a table $R$ (Product, Year, Store, Sales) which could be a base table or an aggregate view of the base table from Level-1 (e.g., Table 1). The following statement will define a summarization on the sales performance of products by different stores in different years using the linguistic terms:

$$
\begin{aligned}
& \text { FUZZ-TERM }\{\text { bad, medium, good }\}\{ \\
& \operatorname{bad}(x)= \begin{cases}-0.004 x+1 & 0 \leq x \leq 250 \\
0 & x>250\end{cases} \\
& \operatorname{medium}(x)= \begin{cases}0.004 x & 0 \leq x \leq 250 \\
-0.004 x+2 & 250<x \leq 500 \\
0 & x>500\end{cases} \\
& \operatorname{good}(x)= \begin{cases}0 & 0<x \leq 250 \\
0.004 x-1 & 250<x \leq 500 \\
1 & x>500\end{cases} \\
& \} \text { ON Sales FROM R }
\end{aligned}
$$

Fig. 7 plots the membership functions defined for the linguistic terms "bad," "medium," and "good" in Example 4.

Note that a linguistic term set must be complete. In other words, it should provide a fuzzy partitioning of the domain of the attribute variable. For example, if the attribute is "age," a linguistic term set could be "young," "middle-aged," or "old." Based on the categorical concepts, we can formally define an $n$-dimensional qualitative data cube.

Definition 2. An n-dimensional qualitative data cube $\mathcal{C}_{c}^{n}$ can be defined as a mapping function $\mathcal{F}_{c}\left(\mathcal{C}_{c}^{n}\right): \operatorname{dom}\left(D_{1}\right) \times$ $\ldots \times \operatorname{dom}\left(D_{n}\right) \rightarrow\{\langle T,[0,1]\rangle\}$, which maps a set of dimensional values to a set of 2-tuples, each indicating a linguistic term and a related membership grade.

Here, $T$ is a complete linguistic term set spanning the domain of the measured attribute. For $\forall t \in T$, a membership function $\mu_{t}$ is defined on scalar values, and $\mu_{t}(N U L L)=0$.

Let $v=\mathcal{F}_{q}\left(\mathcal{C}_{q}^{n}\right)\left(d_{1}, \ldots, d_{n}\right)$ denote a measure in an $n$-dimensional quantitative data cube $\mathcal{C}_{q}^{n}$ at Level-1, where

$$
d_{1} \in \operatorname{dom}\left(D_{1}\right), \ldots, d_{n} \in \operatorname{dom}\left(D_{n}\right),
$$

a corresponding $n$-dimensional qualitative data cube $\mathcal{C}_{c}^{n}$ can be generalized, where

$\mathcal{F}_{c}\left(\mathcal{C}_{c}^{n}\right)\left(d_{1}, \ldots, d_{n}\right)=\left\{<t, \mu_{t}(v)>\mid\left(v=\mathcal{F}_{q}\left(\mathcal{C}_{q}^{n}\right)\left(d_{1}, \ldots, d_{n}\right)\right)\right.$
$\left.\wedge(t \in T) \wedge\left(\mu_{t}(v)>0\right)\right\}$.

Example 5. Table 2 shows a three-dimensional qualitative data cube $\mathcal{C}_{c}^{3}$ at Level-2, which is derived from the quantitative data cube $\mathcal{C}_{q}^{3}$ (Table 1) at Level-1. We use two attributes-"F-Sales" and "F-SalesDegree" to store a tuple $<$ linguistic term, membership grade $>$. An " $F$-" prefix implies a fuzzified attribute. As

$$
v=\mathcal{F}_{q}\left(\mathcal{C}_{q}^{3}\right)(P C, 1997, \text { east })=490,
$$


TABLE 2

Qualitative Summarization Table at Level-2

\begin{tabular}{|c|c|c|c|c|}
\hline \multicolumn{3}{|c|}{ Dimensions } & \multicolumn{2}{|c|}{ Measure } \\
\hline Product & Year & Store & F-Sales & F-SalesDegree \\
\hline $\mathrm{PC}$ & 1997 & east & good/medium & $0.96 / 0.04$ \\
\hline $\mathrm{PC}$ & 1997 & west & medium/bad & $0.8 / 0.2$ \\
\hline $\mathrm{PC}$ & 1998 & south & good/medium & $0.92 / 0.08$ \\
\hline $\mathrm{PC}$ & 1998 & west & good/medium & $0.8 / 0.2$ \\
\hline $\mathrm{PC}$ & 1998 & east & medium/good & $0.8 / 0.2$ \\
\hline $\mathrm{PC}$ & 1998 & north & $\mathrm{bad} /$ medium & $0.8 / 0.2$ \\
\hline Workstation & 1998 & east & good & 1.0 \\
\hline Notcbook & 1998 & cast & good $/$ medium & $0.84 / 0.16$ \\
\hline.. & & & & . \\
\hline
\end{tabular}

and based on the fuzzy term set definition given in Example 4, where

$$
\mu_{\text {good }}(490)=0.96, \mu_{\text {medium }}(490)=0.04, \mu_{\text {bad }}(490)=0,
$$

we have

$$
\begin{aligned}
& \mathcal{F}_{c}\left(\mathcal{C}_{c}^{3}\right)(P C, 1997, \text { east }) \\
& =\left\{<\operatorname{good}, \mu_{\text {good }}(v)>,<\text { medium }, \mu_{\text {medium }}(v)>\right\} \\
& =\{<\operatorname{good}, 0.96>,<\text { medium }, 0.04>
\end{aligned}
$$

\subsubsection{Level-3: Quantifier Summarization}

In addition to the numerical and categorical summarization on each individual measured value, often, business managers want to have some insight into the overall performance of a group of measured values like:

\section{Example 6.}

$Q_{1}^{\prime \prime}:$ "How did most of products' sales behave last year?"

$Q_{2}^{\prime \prime}$ : "What was the average performance of products" sales in the southern area?"

$Q_{3}^{\prime \prime}$ : "At least what percentage of products' sales behavior was good recently?"

Each of the above queries involves two kinds of linguistic terms: ["most," "about average," "at least," and "few" $\}$ and \{"good," "medium," and "bad." $\}$ In the fuzzy area, the former are called linguistic quantifiers [66]. Essentially, linguistic quantifiers can be regarded as fuzzy proportions or fuzzy probabilities. Zadeh represented these linguistic quantifiers as fuzzy sets on the unit interval. The membership grade of any proportion $x \in[0,1], \mu_{q}(x)$, is a measure of the compatibility of the proportion $x$ with the linguistic quantifier represented by the fuzzy set $q$. For example, if $q$ is the quantifier "most," then $\mu_{\text {most }}(0.9)$ represents the degree to which 0.9 satisfies the concept "most" [66].
Linguistic quantifiers actually carry a statistical summary semantics against a set of values. For instance, the statement- "Nearly all the products in 1997 in the east sold well"-makes a summarization against the Product dimension, which we will call a quantified dimension. Similarly, Year is the quantified dimensional attribute in the summary expression "In most of the years, PC sales was good in the west." Furthermore, quantifier summarization can be done over two or more quantified dimensions, like "A few of the products had bad sales in most regions in 1998."

Therefore, if we view the previous Level-2 as a (categorical) fuzzification on the measure of a data cube, Level-3 here is a (quantifier) fuzzification on the dimension $(s)$ of the cube, resulting in possibly smaller dimensional domain(s).

In the same way as linguistic terms, the warehouse administrator can declare a set of linguistic quantifiers of interest through the FUZZ-QUANTIFIER constructor:

FUZZ-QUANTIFIER < linguistic quantifier set >

$<$ membership function set $>$ FOR $<$ quantified attribute $>$

$\mathrm{ON}<$ measurement attribute $>$ FROM $<$ table $>$

Also, a linguistic quantifier set is required to provide a complete fuzzy partitioning of the unit interval [59].

Example 7. Consider a table $R$ (Product, Year, Store, F-Sales, F-SalesDegree) at Level-2. The following statement will quantify the dimensional attribute Product using "most," "around-half," and "few" for the measurement attribute F-Sales.

$$
\begin{aligned}
& \text { FUZZ-QUANTIFIER }\{\text { most, around-half, few }\} \\
& \operatorname{most}(x)= \begin{cases}0 & 0 \leq x \leq 0.5 \\
(2 x-1)^{0.5} & 0.5<x \leq 1.0\end{cases} \\
& \text { around-half }(x)= \begin{cases}1-(1-2 x)^{0.5} & 0 \leq x \leq 0.5 \\
1-(2 x-1)^{0.5} & 0.5<x \leq 1.0\end{cases} \\
& \text { few }(x)= \begin{cases}(1-2 x)^{0.5} & 0 \leq x \leq 0.5 \\
0 & 0.5<x \leq 1.0\end{cases} \\
& \} \text { FOR Product ON F-Sales FROM R }
\end{aligned}
$$

Fig. 8 shows the membership functions defined for the linguistic quantifiers "most," "around-half," and "few" in Example 7.

To conduct quantifier summarization, first, records of the table at Level-2 need to be grouped by all the attributes except the measurement attribute and quantified attributes, and then the third-layered summarization is performed against each group. Hence, one table record at Level-3 actually describes a group of data at Level-2. For instance, each
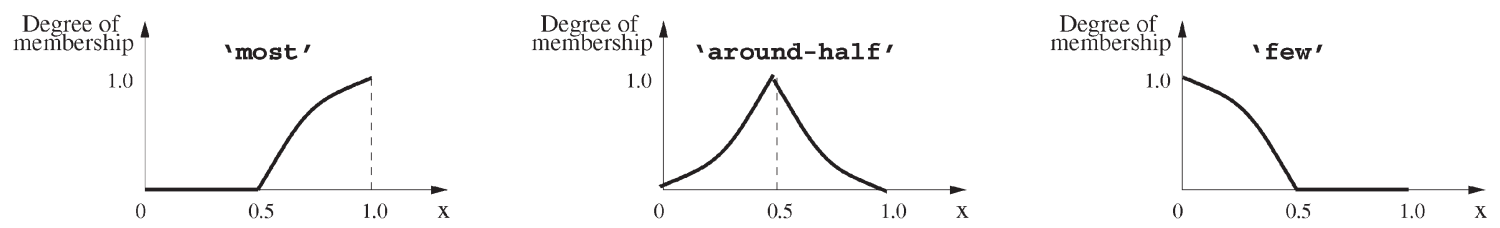

Fig. 8. The membership functions defined in Example 7. 
record resulting from the summarization in Example 7 will deliver an overall product sales performance in a specific year and area. Similarly, we can get the overall performance regarding years for a certain product in a certain region by:

FUZZ-QUANTIFIER \{most, around-half, few $\{$ membership functions can be the same as before $\}$

FOR Year ON F-Sales FROM R

One example summary obtained after executing the above statement is like "In most of the years, PC sold well in the east."

Before giving a formal definition of data cubes at Level-3, we first define a validity function $f(t, G)$ regarding a linguistic term $t$ for a group $G$.

Definition 3. Let $T$ be a complete linguistic term set defined on the measure. Let $Q$ be a complete linguistic quantifier set defined on a quantified dimension $D_{i}$ with $\operatorname{dom}\left(D_{i}\right)=Q \cdot Q$ spans the unit interval $[0,1]$. Let $G\left(d_{1}, \ldots, d_{i-1}, d_{i+1}, \ldots, d_{n}\right)$ denote a group of points in an n-dimensional qualitative data cube at Level-2, each of which has the same dimensional values on $D_{1}, \ldots, D_{i-1}, D_{i+1}, \ldots, D_{n}$ except $D_{i}$. That is, for $\forall g \in G\left(d_{1}, \ldots, d_{i-1}, d_{i+1}, \ldots, d_{n}\right)$, where $g=\left(g_{1}, \ldots, g_{n}\right)$, we have

$$
\begin{aligned}
& \left(g_{1}=d_{1}\right) \wedge \ldots \wedge\left(g_{i-1}=d_{i-1}\right) \wedge\left(g_{i+1}=d_{i+1}\right) \wedge \ldots \\
& \wedge\left(g_{n}=d_{n}\right) \wedge\left(g_{i} \in \operatorname{dom}\left(D_{i}\right)\right) .
\end{aligned}
$$

We use $G$ for $G\left(d_{1}, \ldots, d_{i-1}, d_{i+1}, \ldots, d_{n}\right)$ when the context makes the usage clear, and refer to the total number of points in $G$ as $|G|$.

For $\forall g=\left(d_{1}, \ldots, d_{n}\right) \in G$, let $v_{g}=\mathcal{F}_{q}\left(\mathcal{C}_{q}^{n}\right)\left(d_{1}, \ldots, d_{n}\right)$ denote a quantitative measurement in an n-dimensional quantitative data cube $\mathcal{C}_{q}^{n}$ at Level-1, and

$$
\begin{aligned}
U_{g} & =\mathcal{F}_{c}\left(\mathcal{C}_{c}^{n}\right)\left(d_{1}, \ldots, d_{n}\right) \\
& =\left\{<t, \mu_{t}\left(v_{g}\right)>\right\}\left(t \in T \text { and } \mu_{t}\left(v_{g}\right)>0\right)
\end{aligned}
$$

denote the corresponding qualitative measurement, consisting of a set of 2-tuples for linguistic terms and corresponding membership degrees, at Level-2. A validity function $f(t, G)$ for linguistic term $t \in T$ on group $G$ can be defined as:

$$
\begin{aligned}
& f(t, G)=\sum_{g \in G} \rho\left(t, U_{g}\right) /|G|, \text { where } \\
& \rho\left(t, U_{g}\right)= \begin{cases}\mu_{t}\left(v_{g}\right) & \text { if the tuple }<t, \mu_{t}\left(v_{g}\right)>\in U_{g} \\
0 & \text { otherwise. }\end{cases}
\end{aligned}
$$

As for each member $g \in G, 0 \leq \rho\left(t, U_{g}\right) \leq 1$, the obtained validity function value $f(t, G)$ falls within $[0,1]$ as well.

Example 8. Consider a group $G(1998$, east) consisting of three members $G=\left\{g_{1}, g_{2}, g_{3}\right\}$, where

$g_{1}=(P C, 1998$, east $), g_{2}=($ Workstation, 1998, east $)$,

and

$$
g_{3}=(\text { Notebook, 1998, east). }
$$

The group members share the same dimensional values "Year = 1998" and "Store = east" except the Product dimension in the qualitative data cube in Table 2.

$$
\begin{aligned}
u_{g_{1}} & =\mathcal{F}_{c}\left(\mathcal{C}_{c}^{3}\right)(P C, 1998, \text { east }) \\
& =\{<\text { medium } 0.8>,<\text { good }, 0.2>\} \\
u_{g_{2}} & =\mathcal{F}_{c}\left(\mathcal{C}_{c}^{3}\right)(\text { Workstation, 1998, east }) \\
& =\{<\text { good, } 1.0>\} \\
u_{g_{3}} & =\mathcal{F}_{c}\left(\mathcal{C}_{c}^{3}\right)(\text { Notebook, 1998, east }) \\
& =\{<\text { good }, 0.84>,<\text { medium, } 0.16>\} .
\end{aligned}
$$

The validity function values of the group with respect to linguistic terms-"good," "medium," and "bad" are:

$$
\begin{aligned}
& f(\text { good }, G)=(0.2+1.0+0.84) / 3=0.68, \\
& f(\text { medium }, G)=(0.8+0.0+0.16) / 3=0.32, \\
& f(\text { bad }, G)=(0.0+0.0+0.0) / 3=0.0 .
\end{aligned}
$$

Definition 4. An n-dimensional quantifier data cube $\mathcal{C}_{f}^{n}$ can be defined as a mapping function

$\mathcal{F}_{f}\left(\mathcal{C}_{f}^{n}\right): \operatorname{dom}\left(D_{1}\right) \times \ldots \times \operatorname{dom}\left(D_{n}\right) \rightarrow\{<T,[0,1]>\}$,

which maps a set of dimensional values to a set of 2-tuples, each indicating a linguistic term and a related membership grade from a fuzzy quantifier $q \in Q$, where $Q$ is the domain of the quantified dimension $D_{i}$.

Let $v_{t}=f(t, G)\left(0 \leq v_{t} \leq 1\right)$, and let $\mu_{q}\left(v_{t}\right)$ be the membership grade against the quantifier $q \in Q$. For any $q \in Q$, we use $t^{\prime}$ to represent the linguistic term whose $\mu_{q}\left(v_{t^{\prime}}\right)$ gets the maximum membership grade among all the terms in T, i.e., $\mu_{q}\left(v_{t^{\prime}}\right)=\operatorname{Max}_{t \in T}\left(\mu_{q}\left(v_{t}\right)\right)$. As it is possible to have a set of terms $t_{1}^{\prime}, t_{2}^{\prime}, \ldots, t_{s^{\prime}}^{\prime}$ where

$$
\mu_{q}\left(v_{t_{1}^{\prime}}\right)=\mu_{q}\left(v_{t_{2}^{\prime}}\right)=\ldots=\mu_{q}\left(v_{t_{s}^{\prime}}\right)=\operatorname{Max} \operatorname{MaT}_{t \in T}\left(\mu_{q}\left(v_{t}\right)\right),
$$

an $n$-dimensional quantifier data cube $\mathcal{C}_{f}^{n}$ can be defined, where

$$
\begin{aligned}
& \mathcal{F}_{f}\left(\mathcal{C}_{f}^{n}\right)\left(d_{1}, \ldots, d_{i-1}, q, d_{i+1}, \ldots, d_{n}\right) \\
& =\left\{<t^{\prime}, \mu_{q}\left(v_{t^{\prime}}\right)>\mid\left(t^{\prime} \in T\right) \wedge \mu_{q}\left(v_{t^{\prime}}\right)\right. \\
& \left.=\operatorname{Max}_{t \in T}\left(\mu_{q}\left(v_{t}\right)\right)\right\} .
\end{aligned}
$$

Example 9. Let us perform a quantifier summarization against the group $G(1998$, east $)$ in Example 8. Based on the validity function values,

$v_{\text {good }}=f($ good,$G)=0.68, v_{\text {medium }}=f($ medium,$G)=0.32$,

and

$$
v_{\text {bad }}=f(\text { bad }, G)=0.0,
$$

as well as the FUZZ-QUANTIFIER definition given in Example 7 , we have $\mu_{\text {most }}(0.68)=0.6, \mu_{\text {most }}(0.32)=0.0$, and $\mu_{\text {most }}(0.0)=0.0$. Thus, $t^{\prime}={ }^{\prime \prime}$ good" since it receives the highest membership grade (0.6) compared with the other two terms- "medium" (0.0) and "bad" (0.0). Therefore, $\mathcal{F}_{f}\left(\mathcal{C}_{f}^{3}\right)$ (most, 1998, east $)=\{<$ good, 0.6$\left.\rangle\right\}$.

Table 3 is an example quantifier data cube, where a tuple < linguistic term, quantifier membership grade> are stored by two attributes $Q-F$-Sales and Q-SalesDegree, respectively. A "Q-" prefix denotes the summarized attributes at Level-3.

Similarly, we can compute the measurement value at (few, 1998, east) according to the linguistic quantifier 
TABLE 3

Quantifier Summarization Table at Level-3

\begin{tabular}{|c|c|c|c|c|}
\hline \hline \multicolumn{3}{|c|}{ Dimensions } & \multicolumn{2}{c|}{ Measure } \\
\hline Q-Product & Year & Store & $Q-F-$ Sales & Q-SalesDegree \\
\hline most & 1998 & east & good & 0.6 \\
few & 1998 & east & bad & 1.0 \\
around-half & 1998 & east & good/medium & $0.4 / 0.4$ \\
.. &.. &.. &.. &.. \\
\hline \hline
\end{tabular}

membership function defined for "few" in Example 7, where $f e w(x)=(1-2 x)^{0.5}$ when $0 \leq x \leq 0.5$, and 0 when $0.5<x \leq 1.0$.

Since

$$
\begin{aligned}
& \mu_{\text {few }}\left(v_{\text {good }}\right)=\mu_{\text {few }}(0.68)=0.0, \\
& \mu_{\text {few }}\left(v_{\text {medium }}\right)=\mu_{\text {few }}(0.32)=(1-2 * 0.32)^{0.5}=0.6,
\end{aligned}
$$

and

$$
\mu_{\text {few }}\left(v_{\text {bad }}\right)=\mu_{\text {few }}(0.0)=(1-2 * 0.0)^{0.5}=1.0,
$$

the highest membership degree $\mu_{\text {few }}\left(v_{t^{\prime}}\right)$ of the above three is 1.0 , when $t^{\prime}=$ "bad".

Therefore, $\mathcal{F}_{f}\left(\mathcal{C}_{f}^{3}\right)($ few, 1998, east $)=\{<$ bad, 1.0$\left.\rangle\right\}$.

Note that, all relevant definitions of FUZZ-TERM and FUZZ-QUANTIFIER, together with different-layered summary table information including both logical and physical representations and structures, will enter the enhanced metadata repository to aid users to thoroughly understand the meaning of warehoused data and hence make full use of the data to support their business activities.

\subsection{The Acquisition of Grades of Memberships}

A main problem for the proposed data warehouse semantic model is the choice of appropriate membership functions for different linguistic terms. This is also a major difficulty that has hindered the application of fuzzy techniques in the traditional database field. In this section, we briefly review the traditional membership elicitation approaches, and then explore the problem from the perspective of decision makers under the new data warehouse context. A simple cluster-based strategy is proposed to address this controversial question which has bothered many a scholar for many years.

\subsubsection{The Traditional Approaches}

Several procedures have been suggested in the literature to acquire and construct membership functions. Good surveys can be found in [37], [52], [43]. Basically, there are four methods for the experimental acquisition of membership values, namely, direct rating, polling, set valued statistics, and reverse rating [52].

1. Direct Rating. The membership degrees of elements are assigned by subjects (humans) directly. Usually, the subject is asked to respond to a question like "How $A$ is $x$ ?," where $A$ is the linguistic term, and $x$ is an element in question whose membership degree we seek to acquire. The subject's response is a value $y \in[0,1]$. To alleviate the inherent inconsistencies in human perception, the subject is presented with the same $x$ a reasonable number of times, say $n=10$, and his/her responses are recorded as $y$, i.e., the observed membership value $\mu_{A}(x)$ at a given $x$, from which a conditional distribution function $f(y \mid x)$ can be generated with mean $\bar{y} \mid x=(1 / n) \sum_{i=1}^{n}\left(y_{i} \mid x\right)$ and variance $V(y \mid x)=(n-1)^{-1} \sum_{i=1}^{n}\left(y_{i}|x-\bar{y}| x\right)^{2}$.

2. Polling. Polling is a pure probability-based approach of assigning membership degrees to elements. It randomly and repeatedly presents subjects with an element $x$, and asks for either a "yes" or a "no" response to the question like "Do you agree that $x$ is a member of $A$ ?". The value of $\mu_{A}(x)$ is the probability of respondents answering "yes," i.e.,

$$
\begin{aligned}
& \mu_{A}(x)= \\
& \frac{\text { total number of "yes" responses for } x}{\text { total number of "yes" }+ \text { "no" responses for } x} \text {. }
\end{aligned}
$$

3. Set Valued Statistics. A fuzzy set $A$ can be represented in terms of its level-cuts $\left\{A_{\alpha} \mid \alpha \in[0,1]\right\}$, where $A_{\alpha}=\left\{x \in X \mid \mu_{A}(x) \geq \alpha\right\}$ [18]. Hence, given a random set $R=\left\{\left(A_{\alpha_{i}}, m_{i}\right) \mid i=1, \ldots, n\right\}$, where $A_{\alpha_{i}}$ is a set valued observation on $X$, and $m_{i}$ is the probability that $A_{\alpha_{i}}$ stands as a representative of $A$, the membership degree $\mu_{A}(x)$ can be expressed as

$$
\mu_{A}(x)=\sum_{x \in A_{\alpha_{i}}} m_{i}
$$

4. Reverse Rating. In the reverse rating procedure, randomly selected membership values $y \in[0,1]$ are presented to a subject in a random manner. The subject is asked to respond to the question like "Identify element $x$ that possesses the yth degree of membership in the fuzzy set $A$." The subject's response is an element $x \in X$ for a given membership value $y$.

Once the membership values have been obtained from the experiments, the corresponding membership function curves can then be estimated by the usual regression techniques [47], [52].

\subsubsection{A Cluster-Based Membership Setting Strategy}

Although big progress has been made in the past few years in the acquisition of membership degrees, the lack of simple convincing techniques has often raised criticism to realworld applications of fuzzy set theory. In this study, we reexamine the treatment of fuzziness originating from data warehouse environments, and present a simple clusterbased method, motivated by the following observations. A data warehouse is primarily used by business managers to make enterprise decision. Contrasting with applications which demand precise calculation, the exact numerical value of membership in the interval $[0,1]$ does not matter, but only its order of magnitude under the warehousing context. This is also affirmed by psychological studies demonstrating that a human is normally able to distinguish 

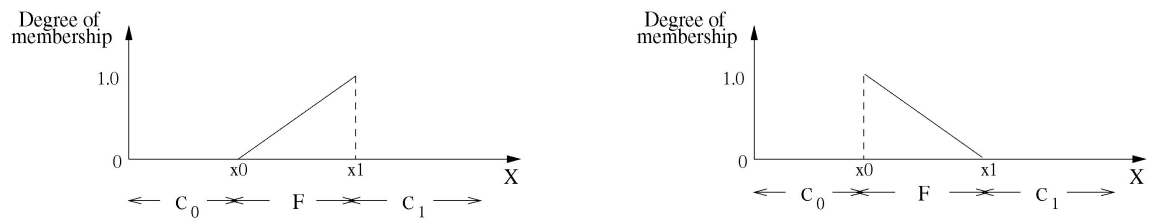

(a)
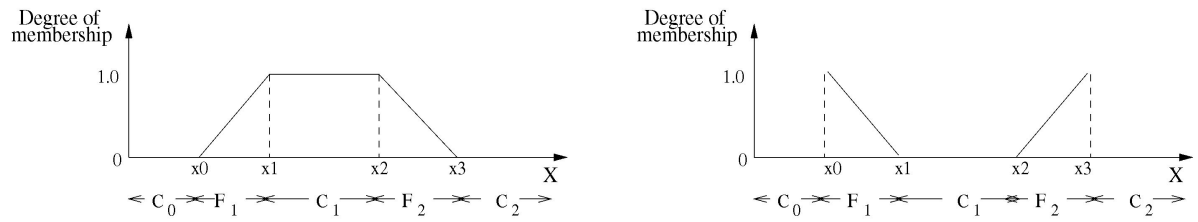

(b)

Fig. 9. (a) Monotonic membership functions and (b) unimodal membership functions.

less than ten degrees of membership only [37]. In the literature, there has been considerable work on fuzzy qualitative reasoning significantly by Dubois et al. (see [15], [14], [17], [3] for good reference).

Hence, in our study, we discretize the unit interval $[0,1]$ into several levels, and measure the membership of elements using these levels rather than precise numbers. To do this, we cluster elements in data warehouses according to their values (e.g., sales amount), so that elements within a cluster are more similar to each other, and thus at the same membership rank than elements in different clusters. The basic procedure for such a clusterbased membership setting method proceeds in three steps.

1. Determine fundamental aspects of membership functions. In the current study, we consider two types of membership functions, i.e., monotonic and unimodal, as shown in Fig. 9. Monotonic membership functions partition $X$ into three regions $C_{0}, F, C_{1}$, while unimodal ones split $X$ into five regions $C_{0}, F_{1}, C_{1}, F_{2}, C_{2}$. Before grouping elements, we need to identify the basic membership shape and relevant regions, which are usually applicationdependent. These can be taken as input parameters whose values are determined by the warehouse administrator according to specific application requirements.

2. Discover clusters of elements. For elements whose values fall into the regions of $C_{0}, C_{1}, C_{2}$, we can easily derive their membership grades-either zero or one. To the rest of elements, we apply the existing PAM clustering algorithm, developed by Kaufman and Rousseeuw [35], to find a set of $k$ element clusters. Here, $k$ can be either given as input by the warehouse administrator, or derived from the elements [35]. We omit the discussion of the $k$-setting issue in this paper.

The basic idea of PAM is to determine a representative object for each cluster. This representative object, called a medoid, is meant to be the most centrally located object within the cluster. Once the medoids have been selected, each nonselected object is grouped with the medoid to which it is the most similar. The quality of a clustering (i.e., the combined quality of the chosen medoids) is measured by the average dissimilarity between an object and the medoid of its cluster. To find the $k$ medoids, PAM begins with an arbitrary selection of $k$ objects. Then, in each step, a swap between a selected object and a nonselected object is made, as long as such a swap would result in an improvement of the quality of the clustering.

Note that, in case of unimodal membership curves as illustrated in Fig. 9b, the clustering process shall be conducted twice: one in the range of $F_{1}$, and another in $F_{2}$.

3. Assign membership ranks to clusters of elements. After identifying the $k$ cluster medoids, we can allocate membership ranks to these clusters according to their relative distances. Take the monotonically increasing membership curve plotted in Fig. 9a for example, suppose that the values of the $k$ cluster medoids are $v_{1}, v_{2}, \ldots, v_{k}$, respectively. We can thus assign membership 0.0 to the elements in $\left[0, x_{0}\right]$, assign membership 1.0 to the elements in $\left[x_{1}, \infty\right]$, and assign membership $\left(v_{i}-x_{0}\right) /\left(x_{1}-x_{0}\right)$ to the elements within the $i$ th cluster, where $1 \leq i \leq k$.

\subsubsection{Remarks}

Compared to the traditional membership acquisition methods, which need substantial experimental inputs from users, the proposed cluster-based strategy incorporates the joint effort from human users and machine. Users indicate the fundamental aspects of membership functions which are application-specific through parameter-settings. The machine then derives the concrete memberships of elements from the data based on the deterministic factors input from users. In this way, the tedious workload on membership assignments can be shifted from humans to the machine, and meanwhile, the subjective nature of linguistic 

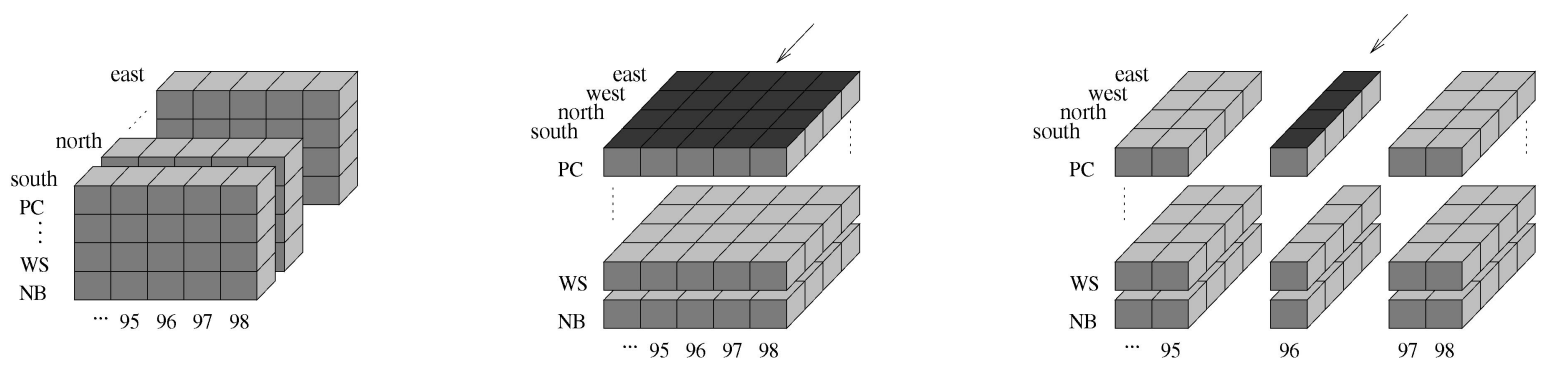

Fig. 10. Different slices of a three-dimensional data cube.

representations can still be guaranteed in the enhanced warehousing systems. By doing this, we can also increase the flexibility of the systems. However, when designing such a warehouse, one could choose to use the cluster-based method or one of the traditional methods discussed above.

Usually, warehouse users are widespread, from headquarters managers, departmental executives and analysts to sales people. Different users, or even the same user in different situations at different times, can view data in different ways. For example, a sales person may think selling three powerful workstations in an industrial region is a perfectly "good" performance, while the general manager in the headquarters may think it is very "poor" in contrast to the sales in other regions where many educational institutes are located. Since it is impossible and also unrealistic to achieve a consistent opinion among different warehouse users, a profile associated with a set of parameter-settings, which describe a subjective view of data (i.e., fundamental aspects of membership functions), can be built for each group of users who share the same opinions. These profiles constitute the contextual environments where different users work with the warehousing system.

\section{Algebraic Operators}

Based on the proposed data model, we now define algebraic operators to facilitate users' access to such enhanced data warehouses. Each of the operators is defined on data cubes and produces as output a new cube. In the following, we use $\mathcal{C}_{s}^{n}(s \in q, c, f)$ to denote an $n$-dimensional data cube, whose type can be quantitative or qualitative or quantifier, depending on the subscript label $s$.

\subsection{Pivot-Oriented Operators}

Pivot-oriented operations include slice, dice, sift, roll-up, and drill-down.

Slice: $\Pi\left(\mathcal{C}_{s}^{n}, D_{1}, \ldots, D_{m}, D_{m+1}=d_{m+1}, \ldots, D_{n}=d_{n}\right)=\mathcal{C}_{s}^{\prime m}$.

The slice operation is used to project data in a data cube on a subset of dimensions for selected values of the other dimensions. Given an input data cube $\mathcal{C}_{s}^{n}$ with $n$ dimensions $D_{1}, D_{2}, \ldots, D_{n}$, assume that $D_{1}, \ldots, D_{m}(m \leq n)$ are the $m$ projected dimensions without loss of generality, and $d_{m+1}, \ldots, d_{n}$ are the selected values of the remaining dimensions $D_{m+1}, \ldots, D_{n}$, from which to slice up the data cube.

The output data cube $\mathcal{C}_{s}^{\prime m}$ being generated has $m$ dimensions $D_{1}, \ldots, D_{m}$. For $\forall\left(d_{1}, \ldots, d_{m}\right)\left(d_{1} \in \operatorname{dom}\left(D_{1}\right) \wedge\right.$ $\left.\ldots \wedge d_{m} \in \operatorname{dom}\left(D_{m}\right)\right)$, we have

$$
\mathcal{F}_{s}\left(\mathcal{C}_{s}^{\prime m}\right)\left(d_{1}, \ldots, d_{m}\right)=\mathcal{F}_{s}\left(\mathcal{C}_{s}^{n}\right)\left(d_{1}, \ldots, d_{m}, d_{m+1}, \ldots, d_{n}\right)
$$

Example 10. Fig. 10 illustrates different slices of the qualitative data cube $\mathcal{C}_{c}^{3}$ in Table 2 by bounding one or two dimensions and letting the rest free. The two arrowindicated slices can be achieved by the operations $\Pi\left(\mathcal{C}_{c}^{3}\right.$, Year, Store, Product $\left.=" P C^{\prime \prime}\right)$ and

$$
\Pi\left(\mathcal{C}_{c}^{3}, \text { Store, Product }=\text { "PC, "Year }=\text { "1996" }\right),
$$

respectively.

Dice: $\sigma\left(\mathcal{C}_{s}^{n}, \mathcal{P}\right)=\mathcal{C}^{\prime n}$. The dice operation is to select data that satisfies a list of predicates connected by logical operators AND, OR, or NOT. A general notion of predicates in our context will be given in the next section. Here, we use $\left\langle d_{1}, \ldots, d_{n}, \mathcal{F}_{s}\left(\mathcal{C}_{s}^{n}\right)\left(d_{1}, \ldots, d_{n}\right)\right\rangle \models \mathcal{P}$ to express that any of attribute values inside this combination of dimensional and measured values satisfies the corresponding requirements posed by $\mathcal{P}$.

Given an input data cube $\mathcal{C}_{s}^{n}$ and a condition $\mathcal{P}$, the dice operation returns the output data cube $\mathcal{C}_{s}^{\prime n}$, where for $\forall\left(d_{1}, \ldots, d_{n}\right)\left(d_{1} \in \operatorname{dom}\left(D_{1}\right) \wedge \ldots \wedge d_{n} \in \operatorname{dom}\left(D_{n}\right)\right)$,

$$
\begin{aligned}
& \left(\mathcal{F}_{s}\left(\mathcal{C}_{s}^{\prime n}\right)\left(d_{1}, \ldots, d_{n}\right)=\mathcal{F}_{s}\left(\mathcal{C}_{s}^{n}\right)\left(d_{1}, \ldots, d_{n}\right)\right) \\
& \wedge\left(\left\langle d_{1}, \ldots, d_{n}, \mathcal{F}_{s}\left(\mathcal{C}_{s}^{n}\right)\left(d_{1}, \ldots, d_{n}\right)\right\rangle \models \mathcal{P}\right) .
\end{aligned}
$$

Note that although the slice $\Pi$ and dice $\sigma$ are both used to get a certain part of data, we define them separately here for two reasons. First, the number of dimensions each delivers is different. The slice operation will reduce the number of dimensions and only keep the projected dimensions in its resulting data cube, while the cube generated by a dice operation maintains all the original dimensions of an input data cube. Second, considering the wide adoption of database storage and query techniques in warehousing systems, we correspond slice and dice to the two fundamental counterpart operators, i.e., projection and selection, in the relational algebra.

Example 11. The expression

$$
\sigma\left(\mathcal{C}_{c}^{3}, \text { Year }=\text { "1996" OR Year }=\right.\text { "1997") }
$$

selects "sales performance of different products sold by different stores in 1996 and 1997" from the cube in Table 2, and delivers a smaller three-dimensional data cube as shown in Fig. 11. 


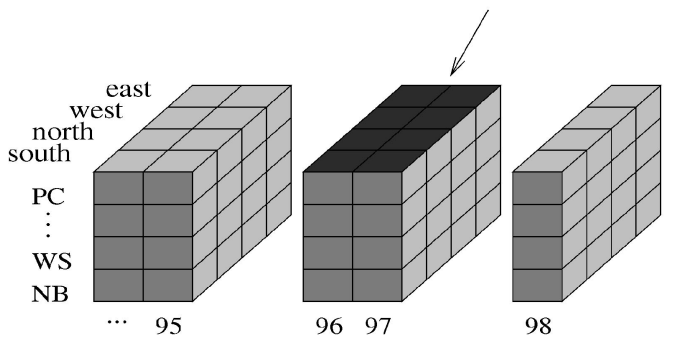

Fig. 11. A dice of a three-dimensional data cube.

Sift: $\varsigma\left(\mathcal{C}_{s}^{n}, r,[t], \ell\right)=\mathcal{C}_{s}^{\prime n}$. The sift operation orders the measured values inside $\mathcal{C}_{s}^{n}$ in the order $\ell \in a s c$, desc, and then outputs $r$ number of the top most measurements into $\mathcal{C}_{s}^{\prime n}$. The ranking of qualitative or quantifier measured values is based on the corresponding membership degrees of the fuzzy term $t$. In the case that $\mathcal{C}_{s}^{n}$ is a traditional quantitative data cube, the parameter $t$ can be omitted. That is, for $\forall\left(d_{1}, \ldots, d_{n}\right)\left(d_{1} \in \operatorname{dom}\left(D_{1}\right) \wedge \ldots \wedge d_{n} \in \operatorname{dom}\left(D_{n}\right)\right)$,

$$
\begin{aligned}
& \mathcal{F}_{s}\left(\mathcal{C}_{s}^{\prime n}\right)\left(d_{1}, \ldots, d_{n}\right)= \\
& \begin{cases}\mathcal{F}_{s}\left(\mathcal{C}_{s}^{n}\right)\left(d_{1}, \ldots, d_{n}\right)=v & \text { if } v \text { is among the } r \text { top most measurements for } t \\
N U L L & \text { otherwise, }\end{cases}
\end{aligned}
$$

where $N U L L$ denotes an empty measure at a certain position in a data cube.

Example 12. The query- "Find the top most three years and stores in which workstations sold well" - can be expressed by a list of operations:

$$
\begin{gathered}
\mathcal{C}_{c}^{\prime 3}=\sigma\left(\mathcal{C}_{c}^{3}, \text { Product }=" \text { Workstation" } A N D\right. \\
F-\text { Sales }=" \text { good" }), \\
\mathcal{C}^{\prime \prime 2}=\Pi\left(\mathcal{C}_{c}^{\prime 3}, \text { Year, Store }\right),
\end{gathered}
$$

and, finally,

$$
\mathcal{C}_{c}^{\prime \prime \prime 2}=\varsigma\left(\mathcal{C}_{c}^{\prime \prime 2}, 3, " \text { good, }, " \text { desc }\right)
$$

Roll-up: $\Uparrow\left(\mathcal{C}_{s}^{n}, D_{1}, \ldots, D_{n-1}, f_{\text {combine }}\right)=\mathcal{C}_{s^{\prime}}^{\prime n-1}$. The roll-up operation combines a group of measured values in $\mathcal{C}_{s}^{n}$ into one based on dimensions $D_{1}, \ldots, D_{n-1}$, which constitute the dimensions of the resulting cube ${\mathcal{C}^{\prime}}_{s^{\prime}}$. A measurement combining function $f_{\text {combine }}$ specifies how these multiple elements are aggregated. It can be either a traditional merging function like SUM or AVG, or an aggregation function defined on a set of fuzzified measurements. In Section 2, we describe some typical conjunctive (or disjunctive) aggregations of elementary matching degrees which can be performed by applying the MIN (or MAX) operation to the fuzzy membership grades. For instance, using MIN for evaluating an aggregation of properties means that the degree of the least satisfied property will reflect the global level of satisfaction. Besides MIN and MAX, more operations like arithmetic MEAN where a low degree of satisfaction for one element can be somewhat compensated by a high degree of satisfaction for another element, or even more drastic arithmetic PRODUCT calculation can be adopted, depending on different application requirements. Here, the input and output data cubes can be of different types, based on the $f_{\text {combine }}$ function being used.
For

$$
\forall\left(d_{1}, \ldots, d_{n-1}\right)\left(d_{1} \in \operatorname{dom}\left(D_{1}\right) \wedge \ldots \wedge d_{n-1} \in \operatorname{dom}\left(D_{n-1}\right)\right),
$$

a group $G\left(d_{1}, \ldots, d_{n-1}\right)$ can be formed in $\mathcal{C}_{s}^{n}, 1$ and, for each point $\left(d_{1}, \ldots, d_{n-1}, d_{n_{i}}\right) \in G$, let $v_{i}=\mathcal{F}_{s}\left(\mathcal{C}_{s}^{n}\right)\left(d_{1}, \ldots, d_{n-1}, d_{n_{i}}\right)$, we have

$$
\mathcal{F}_{s^{\prime}}\left(\mathcal{C}_{s^{\prime}}^{\prime n-1}\right)\left(d_{1}, \ldots, d_{n-1}\right)=f_{\text {combine }}\left(v_{1}, \ldots, v_{|G|}\right) .
$$

Example 13. Assume we have an averaging function on the elementary membership grades of a group-

$$
f_{\text {combine }}\left(v_{1}, \ldots, v_{|G|}\right)=\left(v_{1}+\ldots+v_{|G|}\right) /|G| .
$$

After rolling up the sales data in Table 2 along the dimension Store by $\Uparrow\left(\mathcal{C}_{c}^{3}\right.$, Product, Year, $\left.f_{\text {combine }}\right)$, we obtain a two-dimensional resulting cube $\mathcal{C}^{\prime 2}{ }_{c}$, in which

$$
\begin{aligned}
& \mathcal{F}_{c}\left(\mathcal{C}_{c}^{2}\right)(P C, 1998)=\{<\text { good },(0.92+0.8+0.2+0.0) / 4> \\
& <\text { medium },(0.08+0.2+0.8+0.2) / 4> \\
& <\text { bad },(0.0+0.0+0.0+0.8) / 4>\}=\{<\text { good }, 0.48> \\
& <\text { medium }, 0.32>,<\text { bad }, 0.2>\} .
\end{aligned}
$$

Drill-down: $\Downarrow\left(\mathcal{C}_{s}^{n}, D_{n+1}, f_{\text {split }}\right)=\mathcal{C}_{s^{\prime}}^{\prime n+1}$. The drill-down operation is the converse of roll-up. Function $f_{\text {split }}$ determines the way in which to drill down from an aggregated value to a set of detailed values. $D_{n+1}$ is the added dimension for $\mathcal{C}_{s^{\prime}}^{n+1}$.

Similarly, for

$$
\forall\left(d_{1}, \ldots, d_{n}\right)\left(d_{1} \in \operatorname{dom}\left(D_{1}\right) \wedge \ldots \wedge d_{n} \in \operatorname{dom}\left(D_{n}\right)\right),
$$

a group $G\left(d_{1}, \ldots, d_{n}\right)$ can be formed regarding an $(n+$ 1 )-dimensional data cube, and $|G|=\left|\operatorname{dom}\left(D_{n+1}\right)\right|$.

For $\forall\left(d_{1}, \ldots, d_{n}\right) \quad\left(d_{1} \in \operatorname{dom}\left(D_{1}\right) \wedge \ldots \wedge d_{n} \in \operatorname{dom}\left(D_{n}\right)\right)$, let $v=\mathcal{F}_{s}\left(\mathcal{C}_{s}^{n}\right)\left(d_{1}, \ldots, d_{n}\right)$ and $f_{\text {split }}(v)=v_{1}, \ldots, v_{|G|}$, with each $v_{i}$ corresponding to a new measured value on a certain point $\left(d_{1}, \ldots, d_{n}, d_{n+1_{i}}\right) \in G$, that is,

$$
\mathcal{F}_{s^{\prime}}\left(\mathcal{C}_{s^{\prime}}^{\prime n+1}\right)\left(d_{1}, \ldots, d_{n}, d_{n+1_{i}}\right)=v_{i} .
$$

In order to drill down from a measured value $v$ to its constituents, one has to keep track of how $v$ was obtained. If the merge operation is along the stored path and there is an unique path down the merging tree, then drill-down can be uniquely specified [2].

Example 14. Refer to the rolled-up data cube in Example 13, whose aggregation paths along Store values "east," "north," "south," and "west" for $(P C, 1998)$ have been recorded. The operation $\Downarrow\left(\mathcal{C}^{\prime 2}{ }_{c}\right.$, Store, $\left.f_{\text {split }}\right)$ will get back the three-dimensional data cube $\mathcal{C}_{c}^{3}$ with an extra dimension Store, as shown in Table 2.

\subsection{Measure-Oriented Operators}

Besides the pivot-oriented operators, we also define three measure-oriented operators, i.e., union, intersect, and difference, on two compatible data cubes.

Definition 5. Given two data cubes, $\mathcal{C}_{s}^{n}$ and $\mathcal{C}_{s^{\prime}}^{\prime m}$, with the dimensions $D_{1}, \ldots, D_{n}$ and $D_{1}^{\prime}, \ldots, D_{m}^{\prime}$, respectively, $\mathcal{C}_{s}^{n}$ and

1. Recall that in the previous section, $G$ is used to represent a group of points sharing the same dimensional values except one. 
$\mathcal{C}_{s^{\prime}}^{\prime m}$ are compatible if and only if all the following conditions hold:

1. $\left(s=s^{\prime}\right)$,

2. $(n=m)$, and

3. $\forall i(1 \leq i \leq n)\left(\operatorname{dom}\left(D_{i}\right)=\operatorname{dom}\left(D_{i}^{\prime}\right)\right)$.

Union: $\cup\left(\mathcal{C}_{s}^{n}, \mathcal{C}_{s}^{\prime n}, \oplus\right)=\mathcal{C}_{s}^{\prime \prime n}$. The union operation combines two compatible cubes into one, whose measured values are calculated through the operator $\oplus$. That is, for $\forall\left(d_{1}, \ldots, d_{n}\right) \quad\left(d_{1} \in \operatorname{dom}\left(D_{1}\right) \wedge \ldots \wedge d_{n} \in \operatorname{dom}\left(D_{n}\right)\right)$, let $v=$ $\mathcal{F}_{s}\left(\mathcal{C}_{s}^{n}\right)\left(d_{1}, \ldots, d_{n}\right)$ and $v^{\prime}=\mathcal{F}_{s}\left(\mathcal{C}_{s}^{\prime n}\right)\left(d_{1}, \ldots, d_{n}\right)$, we have

$$
\mathcal{F}_{s}\left(\mathcal{C}_{s}^{\prime \prime n}\right)\left(d_{1}, \ldots, d_{n}\right)=v \oplus v^{\prime}
$$

Intersect: $\cap\left(\mathcal{C}_{s}^{n}, \mathcal{C}_{s}^{\prime n}, \otimes\right)=\mathcal{C}_{s}^{\prime \prime n}$. Similarly, the operator $\otimes$ is used to calculate the intersection of two measured values from the input compatible data cubes. That is, for $\forall\left(d_{1}, \ldots, d_{n}\right) \quad\left(d_{1} \in \operatorname{dom}\left(D_{1}\right) \wedge \ldots \wedge d_{n} \in \operatorname{dom}\left(D_{n}\right)\right)$, let $v=$ $\mathcal{F}_{s}\left(\mathcal{C}_{s}^{n}\right)\left(d_{1}, \ldots, d_{n}\right)$ and $v^{\prime}=\mathcal{F}_{s}\left(\mathcal{C}_{s}^{\prime n}\right)\left(d_{1}, \ldots, d_{n}\right)$, we have

$$
\mathcal{F}_{s}\left(\mathcal{C}_{s}^{n \prime \prime}\right)\left(d_{1}, \ldots, d_{n}\right)=v \otimes v^{\prime} .
$$

Difference: $-\left(\mathcal{C}_{s}^{n}, \mathcal{C}_{s}^{\prime n}, \ominus\right)=\mathcal{C}_{s}^{n \prime \prime}$. The difference between two compatible cubes leads to another compatible one, whose measured values are obtained through the operation $\ominus$. That is, for $\forall\left(d_{1}, \ldots, d_{n}\right)\left(d_{1} \in \operatorname{dom}\left(D_{1}\right) \wedge \ldots \wedge d_{n} \in \operatorname{dom}\left(D_{n}\right)\right)$, let $v=\mathcal{F}_{s}\left(\mathcal{C}_{s}^{n}\right)\left(d_{1}, \ldots, d_{n}\right)$ and $v^{\prime}=\mathcal{F}_{s}\left(\mathcal{C}_{s}^{\prime n}\right)\left(d_{1}, \ldots, d_{n}\right)$, we have

$$
\mathcal{F}_{s}\left(\mathcal{C}_{s}^{\prime \prime n}\right)\left(d_{1}, \ldots, d_{n}\right)=v \ominus v^{\prime}
$$

The semantics of the above $\oplus, \otimes$, and $\ominus$ operators vary with particular warehousing applications. In the presence of qualitative or quantifier data cubes, we can apply a variety of well developed fuzzy set operations like the commonly used UNION, INTERSECT, and DIFFERENCE (as described in Section 2) to the data cube computations.

Example 15. Assume that $\mathcal{C}_{c}^{2}$ and ${\mathcal{C}^{\prime}}_{c}^{2}$ are two qualitative data cubes, built by two different departmental stores. Each cube records yearly sales of different products like

$$
\begin{aligned}
& \mathcal{F}_{c}\left(\mathcal{C}_{c}^{2}\right)(P C, 1998)=\{<\text { good }, 0.6>,<\text { medium }, 0.4>\}, \\
& \mathcal{F}_{c}\left(\mathcal{C}_{c}^{\prime 2}\right)(P C, 1998)=\{<\text { good }, 0.9>\} .
\end{aligned}
$$

Suppose the headquarters of the chain store wants to have an optimistic (respectively, pessimistic) view of sales performance, a MAX (MIN) operation can be applied to union (respectively, intersect) these two cubes:

$$
\cup\left(\mathcal{C}_{c}^{2}, \mathcal{C}_{c}^{\prime 2}, \mathrm{MAX}\right)=\mathcal{C}_{c}^{\prime \prime 2} \text { and } \cap\left(\mathcal{C}_{c}^{2}, \mathcal{C}_{c}^{\prime 2}, \mathrm{MIN}\right)=\mathcal{C}_{c}^{\prime \prime \prime 2},
$$

where

$$
\begin{aligned}
& \mathcal{F}_{c}\left(\mathcal{C}_{c}^{\prime \prime 2}\right)(P C, 1998) \\
& =\{<\operatorname{good}, \operatorname{MAX}(0.6,0.9)>,<\text { medium, } \operatorname{MAX}(0.4,0.0)>\} \\
& =\{<\text { good, } 0.9>,<\text { medium }, 0.4>\} \\
& \mathcal{F}_{c}\left(\mathcal{C}_{c}^{\prime \prime \prime 2}\right)(P C, 1998) \\
& =\{<\text { good, } \operatorname{MIN}(0.6,0.9)>,<\text { medium, } \operatorname{MIN}(0.4,0.0)>\} \\
& =\{<\text { good, } 0.6>,<\text { medium }, 0.0>\} .
\end{aligned}
$$

\section{Queries Against the Enhanced Data WAREHOUSES}

In Section 4, we described a set of algebraic operations on the enhanced multilayered warehouses. These operators are procedural, and users need to specify how-in what order-to apply and execute the operations. In this section, we propose an extension of SQL, a well known nonprocedural query language, by incorporating linguistic terms and quantifiers into users' queries. We have chosen to extend SQL so as not to stray too far from the form of queries currently used by the relational database community.

\subsection{Extension of SQL Query Language}

Following exactly the same philosophy as SQL (as to querying features and syntax in particular) [40], the basic block of the extended query language has the following constructs, with the clauses between [...] being optional:

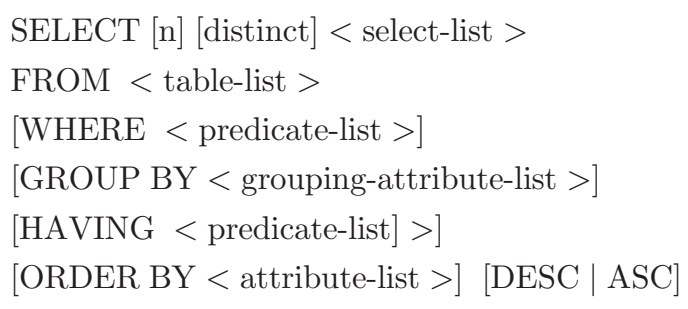

- $\quad$ The $<$ select-list $>$ in the SELECT clause consists of a list of 1) attribute names and 2) terms having the form aggop (attribute name). Any of the aggregation operators in count, sum, max, min, avg can be used for aggop.

$$
\begin{aligned}
& <\text { attribute] }>::= \\
& <\text { normal attribute }>\mid<\text { fuzzified attribute }>\mid \\
& <\text { quantified attribute }>\mid<\text { membership attribute }>
\end{aligned}
$$

For example, the previously defined F-Sales is a fuzzified attribute, $Q$-Product is a quantified attribute, and F-SalesDegree and Q-SalesDegree belong to the membership attributes. They are treated in the same way as the normal attributes within an SQL statement.

- The FROM clause specifies all tables needed in a query.

- The WHERE clause specifies the conditions for selection of records from the table(s). The <predicatelist $>$ expresses a list of predicates connected by logical connectives AND, OR, or NOT. Here, predicates fall into two categories: normal predicates and enhanced predicates. A normal predicate is of the form expression op expression, where op is one of the normal comparison operators in $\{<, \leq,=, \neq,>, \geq\}$. An expression can be an attribute name, a constant, or an arithmetic or string expression. Linguistic terms and quantifiers like "good, (bad)" and "most $(f e w)^{\prime \prime}$ can also be viewed as a kind of string constants, which we call linguistic constants in the following: 


$$
\begin{aligned}
&<\text { enhanced predicate }>::=<\text { attribute }>[\mathrm{mod}]^{*} \\
&<\text { op }_{e}>[\mathrm{mod}]^{*} \\
&<\text { linguistic constant }> \\
& {[(\text { SATISFY }=v)] \mid } \\
&<\text { attribute }>[\text { mod }]^{*} \\
&<\text { op }_{e}><\text { attribute }> \\
& {[\text { SATISFY }=v)] } \\
&<\text { op }_{e}>::=<\text { normal comparison operator }>\mid \\
&<\text { fuzzy comparison operator }> \\
&<\text { linguistic constant }>::=<\text { linguistic term }>\mid \\
&<\text { linguistic quantifier }>
\end{aligned}
$$

- $\quad[\mathbf{m o d}]^{*}$ represents a list of modifiers, prefixing a fuzzified or quantified attribute or linguistic constant, to model the effect of linguistic hedges. For instance, the commonly used modifiers like "very," "much," "rather," "more-or-less," and "approximately" can be linked with "good" to concentrate or dilate the membership function of the fuzzy set "good" (Section 2). Each time none or $k$ number of modifiers (e.g., "very, very

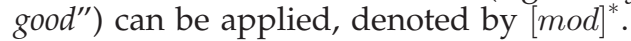

Linguistic modifiers enrich the query vocabulary by deriving new fuzzy sets in terms of previously defined sets, whilst the warehouse manager only needs to care for the most concise linguistic labels.

- To support flexible queries so as to provide a reply while a classical query returns an empty response because it is too stringent, a set of fuzzy comparison operators (e.g., "close to," "similar," "around," "approximate," etc.) are introduced to express the conditions like "This year's sales is close to the last year's." The linguistic modifiers discussed above can also be associated with fuzzy comparison operators to express different satisfactory degrees (e.g., "very close to").

For each fuzzy comparison operator, there is a corresponding membership function defined by the warehouse administrator through the FUZZ-COMPARATOR construct.

\section{FUZZ-COMPARATOR \\ $<$ fuzzy comparison operator $>$ \\ $<$ membership function $>$}

Example 16. An example definition for the "close-to" operator is like:

FUZZ-COMPARATOR close-to \{

(1) $x, y \in$ Numeric Domain

close-to $(x, y)=e^{-\beta|x-y|}$, where $\beta>0$

(2) $x, y \in$ Fuzzy Domain

close-to $(x, y)= \begin{cases}e^{-\beta\left|x \cdot \mu_{x}-\left(y \cdot \mu_{y}\right)^{n}\right|} & \text { if } x=[\bmod ]^{*} y \\ 0 & \text { otherwise }\end{cases}$

\} $x . \mu_{x}$ and $y . \mu_{y}$ represent the membership grades of fuzzy term $x$ and $y$, respectively. When there exist some modifiers as a bridge between the fuzzy terms $x$ and $y$ (e.g., $x=$ "very good" and $y=$ "good"), the most common modifier function is applied to the modified fuzzy term membership (e.g., $\left.y . \mu_{y}^{n}\right)$, where $n=2$ for a concentrator and $n=0.5$ for a dilator as described in Section 2 .

Fuzzy comparison operators extend the expressive power of the query language. ${ }^{2}$ As they can simulate a human's approximate reasoning, it is very useful and also important to integrate them together with fuzzy terms to aid human's decision-making.

- When a query predicate involves the use of linguistic modifiers and/or a fuzzy comparison operator, a membership grade representing the matching degree value between the enhanced predicate and each record will be calculated and returned. We use the clause (SATISFY $=v$ ), where $v \in[0,1]$ to specify the satisfactory requirement $(\geq v)$. The default value of $v$ is 1.0 .

Each enhanced predicate can be enforced by a satisfactory degree $v$ in a query. In the presence of a compound condition comprised of several enhanced predicates, these values can be used to indicate different weights of importance of the corresponding predicate.

Note that the satisfactory degree $v$ in the SATISFY clause is only applicable to the enhanced predicates containing fuzzy comparison operators and/or modifiers, whose matching degree with the record needs to be dynamically calculated during the query execution. This is different from the membership attributes (e.g., F-SalesDegree, Q-SalesDegree) whose grade values (e.g., $\left.\mu_{\text {bad }}(r), \mu_{\text {most }}\left(v_{t}\right)\right)$ have been obtained and stored statically in the data warehouse already when conducting different levels of summarization. For the latter, we can directly use a normal predicate (e.g., F-SalesDegree $\geq \alpha$ ) to express an $\alpha$-cut requirement.

- GROUP BY specifies grouping attributes, whereas HAVING specifies a condition on the groups being selected.

- The parameter $\mathbf{n}$ in the SELECT clause specifies the size of the answer, i.e., the number of desired response records. Its appearance must always be accompanied by the ORDER BY clause, indicating only the top most $n$ number of sorted records should be returned. For example, we can rank-order the result records of good sales products according to their membership grades from the highest to the lowest through "ORDER BY F-SalesDegree DESC" statement. The keyword ASC can be used to specify an ascending order.

\subsection{Query Examples}

In this section, we illustrate the usage of the query constructors through a set of queries. Consider two

2. The definition of each fuzzy comparison operator will be recorded by the metadata repository so as to provide business clients with a complete awareness of query facilities in the warehouse. 
summary tables R1 and R2 from Level-2 and Level-3, respectively:

$R 1$ (Product, Year, Store, F-Sales, F-SalesDegree) $R 2$ (Q-Product, Year, Store, Q-F-Sales, Q-SalesDegree).

Query 1. Find two top most products whose sales performance in the east was good in 1998.

\section{SELECT $\quad 2$ Product FROM $R 1$ \\ WHERE Store $=$ "east" AND Year $=$ "1998" AND$$
\text { F-Sales = "good" }
$$ \\ ORDER BY F-SalesDegree DESC}

The execution of this query will return two products whose membership degrees of "good" are ranked among the top two in the east in 1998. Assume the records of table $R 1$ are within Table 2, from which three products (i.e., $P C$, Workstation, and Notebook) were sold well (with membership grades $0.2,1.0$, and 0.84 , respectively) in the east in 1998. The above query will return two products Workstation and Notebook, since their membership grades are among the top two.

Query 2. Find yearly sales performance of PC in the east (with $\alpha$-cut $=0.8$ ).

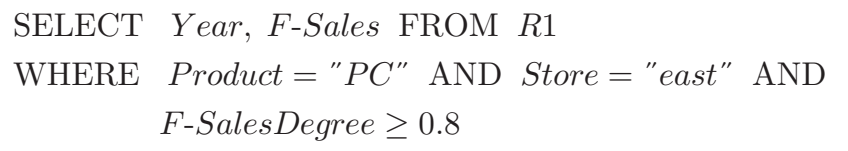

Fuzzified attributes can also be used within the GROUP BY and HAVING clauses.

Query 3. Find the three best years and regions in which workstations were sold well.

$\begin{array}{ll}\text { SELECT } & 3 \text { Year, Store FROM } R 1 \\ \text { GROUP BY } & \text { Year, Store } \\ \text { HAVING } & \text { Product }=\text { "Workstation"AND } \\ & \text { F-Sales }=\text { "good" } \\ \text { ORDER BY } & \text { F-SalesDegree DESC }\end{array}$

Traditional aggregate operations can be applied to fuzzy membership attributes which are treated as normal numerical attributes.

Query 4. Find bad sales products and their average membership grades in 1998.

$\begin{array}{ll}\text { SELECT } & \text { Product, AVG(F-SalesDegree }) \text { FROM } R 1 \\ \text { WHERE } & \text { Year }=" 1998 " \\ \text { GROUP BY } & \text { F-Sales } \\ \text { HAVING } & \text { F-Sales }=" \text { bad" }\end{array}$

In addition, users can retrieve the overall behavior of products via queries like:

Query 5. Find years in which most products' sales performance was bad.
SELECT Year FROM $R 2$

WHERE Q-product $=$ "most" AND Q-F-Sales $="$ bad"

Query 6. Find the overall sales situation of products in the west.

SELECT Q-Product, Q-F-Sales FROM $R 2$

WHERE Store $=$ "west"

An enhanced predicate query, containing linguistic modifiers or fuzzy comparison operators, is usually accompanied by the SATISFY clause as below:

Query 7. Find the years in which PC sales were very good (the satisfaction degree is 0.8 ).

SELECT Year FROM $R 1$

WHERE Product $=$ "PC" AND F-Sales = "very" "good" $(\mathrm{SATISFY}=0.8)$

Suppose $r$ is a record in table $R 1$ with r.Product $=$ "PC," r.F-Sales $=$ "good," and r.F-SalesDegree $=a$. Prefixed with the modifier "very," its membership degree regarding "very good" becomes $a^{2}$. Apparently, the first predicate is fully satisfied with a grade 1.0. The record $r$ will be returned only if $\operatorname{MIN}\left(1.0, a^{2}\right)=a^{2} \geq 0.8$.

Query 8. Find products which had similar sales behavior in the past two years (the satisfaction degree is 0.9).

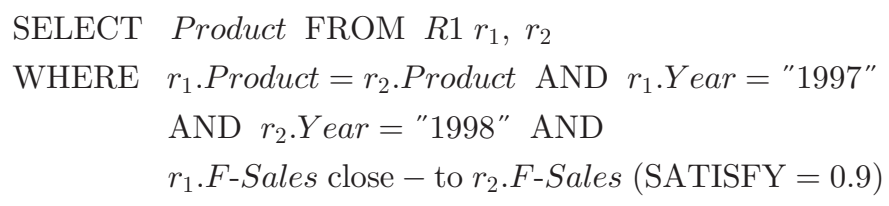

The membership function of "close-to" will return a grade value concerning a product's sales difference in the past two years, which is requested to be equal or greater than 0.9 in this query.

\section{Conclusion}

In this paper, we explore the use of fuzzy technology to provide semantics to the data in data warehouses. A threelayered data warehouse semantic model consisting of quantitative (numerical) summarization, qualitative (categorical) summarization, and quantifier summarization was proposed. Traditional aggregate views in data warehouses constitute the bottom quantitative (numerical) summarization level. Further summarization upon these numerical summary data in terms of linguistic terms forms the middle qualitative (categorical) summarization level. The upper quantifier summarization level provides an overall picture about a set of records through linguistic quantifiers like "most," "around half," "few," etc. In order to facilitate the construction, operation and querying of such enhanced data warehouses, several constructors and operators are introduced in this paper.

We view this work as a first step with a number of interesting problems and opportunities remaining for future work. First, to achieve a more powerful and flexible decision support, efficient storage of summary data at 
different abstract levels, as well as the enhanced metadata repository, is important and must be carefully planned. Beyond quantifier summarization, more data analysis and data mining results can be incorporated, leading to higher summarization levels and association (classification) rules. Further, it is also interesting to investigate different uninorm and quantifier-guided aggregations which are well-developed in the fuzzy area [61], [58], [60], [57] in a data warehousing environment. Currently, we are conducting research into the development of a physical data model and efficient implementation strategies for the proposed data warehouse semantic model.

\section{ACKNOWLEDGMENTS}

The authors would like to thank the anonymous reviewers for valuable comments and suggestions that have greatly improved the quality of this paper. This work was done while Ling Feng was in the Infolab, Tilburg University, Netherlands.

\section{REFERENCES}

[1] S. Agarwal, R. Agrawal, P. Deshpande, A. Gupta, J.F. Naughton, R. Ramakrishnan, and S. Sarawagi, "On the Computation of Multidimensional Aggregates," Proc. 22th Int'l Conf. Very Large Data Bases, Sept. 1996.

[2] R. Agrawal, A. Gupta, and S. Sarawagi, "Modeling Multidimensional Databases," Proc. Int'l Conf. Data Eng., 1997.

[3] N.B. Amor, S. Benferhat, D. Dubois, H. Geffner, and H. Prade, "Independence in Qualitative Uncertainty Frameworks," Proc. Seventh Int'l Conf. Principles of Knowledge Representation and Reasoning, pp. 235-246, Apr. 2000.

[4] P. Bosc, "Some Approaches for Processing SQLf Nested Queries," Intelligent Systems, vol. 11, no. 9, pp. 65-74, Sept. 1996.

[5] P. Bosc, F. Connan, and D. Rocacher, "Flexible Querying in Multimedia Databases with an Object Query Language," Proc. Seventh IEEE Int'l Conf. Fuzzy Systems, pp. 1308-1313, May 1998.

[6] P. Bosc and O. Pivert, "SQLf: A Relational Database Language from Fuzzy Querying," IEEE Trans. Fuzzy Systems, vol. 3, no. 1, pp. 1-17, 1995.

[7] L. Cabibbo and R. Torlone, "From a Procedural to a Visual Query Language for OLAP," Proc. IEEE Int'l Conf. Very Large Data Bases, 1996.

[8] L. Cabibbo and R. Torlone, "A Framework for the Investigation of Aggregate Functions in Database Queries," Proc. Seventh Int'l Conf. Database Theory, 1999.

[9] S. Chaudhuri and U. Dayal, "An Overview of Data Warehousing and OLAP Technology," SIGMOD Record, vol. 26, no. 1, pp. 65-74, 1997.

[10] E. Cox, The Fuzzy Systems Handbook. Academic Press, Inc., 1994.

[11] S. Dar, H.V. Jagadish, A.V. Levy, and D. Srivastava, "Answering Queries with Aggregation Using Views," Proc. 22th Int'l Conf. Very Large Data Bases, 1996.

[12] C.R. De, "Fuzzy and Uncertain Object-Oriented Databases: Concepts and Models." World Scientifc, 1997.

[13] P. Deshpande, K. Ramasamy, A. Shukla, and J.F. Naughton, “Caching Multidimensional Queries Using Chunks," Proc. ACM SIGMOD Int'l Conf. Management of Data, pp. 271-282, 1998.

[14] D. Dubois, D.L. Berre, H. Prade, and R. Sabbadin, "Logical Representations and Computation of Optimal Decisions in a Qualitative Setting," Proc. 15th Int'l Conf. Artificial Intelligence and 10th Innovative Applications of Artificial Intelligence, pp. 588-593, July 1988.

[15] D. Dubois, D.L. Berre, H. Prade, and R. Sabbadin, "Using Possibilistic Logic for Modeling Qualitative Decision: ATMSBased Algorithms," Fundamental Informaticae, vol. 37, no. 1-2, pp. 130, 1999.

[16] D. Dubois, F. Esteva, P. Garcia, L. Godo, D.M.R. Lopez, and H. Prade, "Fuzzy Set Modeling in Case-Based Reasoning," Intelligent Systems, vol. 13, pp. 301-374, 1996.
[17] D. Dubois, L. Godo, H. Prade, and A. Zapico, “Making Decisions in a Qualitative Setting: From Decision Under Uncertainty to Case-Based Decision," Proc. Fifth Int'l Conf. Principles of Knowledge Representation and Reasoning, pp. 594-607, June 1998.

[18] D. Dubois and H. Prade, "Fuzzy Sets, Probability and Measurement," European J. Operational Research, vol. 40, pp. 135-154, 1989.

[19] D. Dubois and H. Prade, Fuzzy Set and Systems: Theory and Applications. Academic Press, Inc., 1994.

[20] M. Fang, N. Shivakumar, H. Garcia-Molina, R. Motwani, and J.D. Ullman, "Computing Iceberg Queries Efficiently," Proc. 24th Int'l Conf. Very Large Data Bases, pp. 299-310, Aug. 1998.

[21] H. Garcia-Molina, W. Labio, and J. Yang, "Expiring Data in a Warehouse," Proc. 23rd Int'l Conf. Very Large Data Bases, Aug. 1998.

[22] S.R. Gardner, "Building the Data Warehouse," IEEE Trans. Systems, Man, and Cybernetics, vol. 41, no. 9, pp. 52-60, Sept. 1998.

[23] F. Gingras and L.V.S. Lakshmanan, "nD-SQL: A Multi-Dimensional Language for Interoperability and OLAP," Proc. 23rd Int'l Conf. Very Large Data Bases, Aug. 1998.

[24] J. Gray, A. Bosworth, A. Layman, and H. Pirahesh, "Data Cube: A Relational Aggregation Operator Generalizing Group-By, CrossTab, and Sub-Total," Proc. Int'l Conf. Data Eng., Feb. 1996.

[25] T. Griffin and L. Libkin, "Incremental Maintenance of Views with Duplicates," Proc. ACM SIGMOD Int'l Conf. Management of Data, May 1995.

[26] A. Gupta, I.S. Mumick, and K.A. Ross, "Adapting Materialized Views After Redefinitions," Proc. ACM SIGMOD Int'l Conf. Management of Data, May 1995.

[27] H. Gupta, "Selection of Views to Materialize in a Data Warehouse," Proc. Int'l Conf. Database Theory, Jan. 1997.

[28] H. Gupta, "Selection of Views to Materialize in a Data Warehouse," Proc. 23rd Int'l Conf. Very Large Data Bases, 1997.

[29] M.S. Hacid, P. Marcel, and C. Rigotti, "A Rule Based CQL for 2Dimensional Tables," Proc. Second Int'l Workshop Constraint Database Systems, pp. 92-104, Jan. 1997.

[30] M.S. Hacid, P. Marcel, and C. Rigotti, "A Rule Based Data Manipulation Language for OLAP Systems," Proc. Fifth Int'l Conf. Deductive and Object-Oriented Databases, Dec. 1997.

[31] V. Harinarayan, A. Rajaraman, and J. Ullman, "Implementing Data Cubes Efficiently," Proc. ACM SIGMOD Int'l Conf. Management of Data, June 1996.

[32] N. Huyn, "Multiple-View Self-Maintenance in Data Warehousing Environments," Proc. 23rd Int'l Conf. Very Large Data Bases, 1997.

[33] H.V. Jagadish, P.P.S. Narayan, S. Seshadri, and R. Kanneganti, "Incremental Organization for Data Recording and Warehousing," Proc. 23rd Int'l Conf. Very Large Data Bases, pp. 16-25, 1997.

[34] T. Johnson and D. Shasha, "Some Approaches to Index Design for Cube Forests," IEEE Data Eng. Bull., vol. 20, no. 1, pp. 27-35, Mar. 1997.

[35] L. Kaufman and P.J. Rousseeuw, Finding Groups in Data: An Introduction to Cluster Analysis. John Wiley \& Sons, 1990.

[36] G.J. Klir and T.A. Folger, Fuzzy Sets, Uncertainty and Information. Prentice Hall, Inc., 1988.

[37] R. Kruse, J. Gebhardt, and F. Klawonn, Foundations of Fuzzy Systems. John Wiley \& Sons, 1994.

[38] W. Labio, D. Quass, and B. Adelberg, "Physical Database Design for Data Warehousing," Proc. Int'l Conf. Data Eng., 1997.

[39] L. Libkin, R. Machlin, and L. Wong, "A Query Language for Multidimensional Arrays: Design, Implementation, and Optimization Techniques," Proc. ACM SIGMOD Int'l Conf. Management of Data, June 1996.

[40] P. Bosc and H. Prade, "Uncertainty Management in Information Systems: From Needs to Solutions," An Introduction to Fuzzy Sets and Possibility Theory-based Treatment of Soft Queries and Uncertain or Imprecise Databases, Kluwer Academic Publisher, Inc., A. Motro and P. Smets, eds., 1997.

[41] I.S. Mumick, D. Quass, and B.S. Mumick, "Maintenance of Data Cubes and Summary Tables in a Warehouse," Proc. ACM SIGMOD Int'l Conf. Management of Data, pp. 100-111, June 1997.

[42] H. Nakajima and Y. Senoh, "A Spreadsheet-Based Fuzzy Retrieval System," Intelligent Systems, vol. 11, no. 9, pp. 661-670, Sept. 1996.

[43] A.M. Norwich and I.B. Turksen, "A Model for the Measurement of Membership and the Consequences of Its Empirical Implementation," Fuzzy Sets and Systems, vol. 12, pp. 1-25, 1984.

[44] P. O'Neil and D. Quass, "Improved Query Performance with Variant Indexes," Proc. ACM SIGMOD Int'l Conf. Management of Data, pp. 38-49, June 1997. 
[45] F.E. Petry, Fuzzy Databases: Principles and Applications. Kluwer Academic Press, Inc., 1996.

[46] D. Quass and J. Widom, “On-Line Warehouse View Maintenance for Batch Updates," Proc. ACM SIGMOD Int'l Conf. Management of Data, pp. 393-404, June 1997.

[47] P. Zysno, "Empirical Semantics," Modeling Membership Functions, B. Rieger, ed., vol. 1, pp. 350-375, 1981.

[48] K.A. Ross, D. Srivastava, and D. Chatziantoniou, "Complex Aggregation at Multiple Granularities," Proc. Int'l Conf. Extended Database Technology, Apr. 1998.

[49] G. Roy, E.P. Frederick, P.B. Bill, and S. Radhakrishnan, "Fuzzy Database Systems-Challenges and Opportunities of a New Era," Intelligent Systems, vol. 11, no. 9, pp. 649-659, Sept. 1996.

[50] A. Shukla, P. Deshpande, J.F. Naughton, and K. Ramaswamy, "Storage Estimation for Multidimensional Aggregates in the Presence of Hierarchies," Proc. 22nd Int'l Conf. Very Large Data Bases, Sept. 1996.

[51] T. Terano, K. Asai, and M. Sugeno, Fuzzy Set Theory and Its Applications. Academic Press, Inc., 1991.

[52] I.B. Turksen, "Measurement of Membership Functions and Their Acquisition," Fuzzy Sets and Systems, vol. 40, pp. 5-38, 1991.

[53] J. Widom, "Research Problems in Data Warehousing," Proc. Int'l Conf. Information and Knowledge Management, 1995.

[54] M.C. Wu and A.P. Buchmann, "Research Issues in Data Warehousing," Technical Report BTW, Ulm, Mar. 1997.

[55] M.C. Wu and A.P. Buchmann, "Encoded Bitmap Indexing for Data Warehouses," Proc. Int'l Conf. Data Eng., pp. 220-230, 1998.

[56] R.R. Yager, "Quantifiers in the Formulation of Multiple Objective Decision Functions," Information Science, vol. 31, pp. 107-139, 1983.

[57] R.R. Yager, "Aggregation Operators and Fuzzy Systems Modeling," Fuzzy Sets and Systems, vol. 67, pp. 129-146, 1995.

[58] R.R. Yager, "A Unified Approach to Aggregation Based on MOM and MAM Operators," Intelligent Systems, vol. 10, pp. 809-855, 1995.

[59] R.R. Yager, "Database Discovery Using Fuzzy Sets," Intelligent Systems, vol. 11, no. 9, pp. 691-712, Sept. 1996

[60] R.R. Yager, "Quantifier Guided Aggregation Using OWA Operators," Intelligent Systems, vol. 11, pp. 49-73, 1996.

[61] R.R. Yager and A. Rybalov, "Uninorm Aggregation Operators," Fuzzy Sets and Systems, vol. 80, pp. 111-120, 1996.

[62] J. Yang, K. Karlapalem, and Q. Li., "A Framework for Designing Materialized Views in Data Warehousing Environment," Proc. Int'l Conf. Distributed Computing Systems, May 1997.

[63] J. Yang and J. Widom, "Maintaining Temporal Views over Nonhistorical Information Sources for Data Warehousing," Proc. Int'l Conf. Data Eng., 1998.

[64] L.A. Zadeh, "Fuzzy Sets," Information and Control, vol. 8, pp. 338353, 1965.

[65] L.A. Zadeh, "Outline of a New Approach to the Analysis of Complex Systems and Decision Processes," IEEE Trans. Systems, Man, and Cybernetics, vol. 3, no. 1, 1973.

[66] L.A. Zadeh, "A Computational Approach to Fuzzy Quantifiers in Natural Languages," Comput. Math. Applications, vol. 9, pp. 149184, 1983.

[67] Y. Zhao, P.M. Deshpande, J.F. Naughton, and A. Shukla, "Simultaneous Optimization and Evaluation of Multiple Dimensional Queries," Proc. ACM SIGMOD Int'l Conf. Management of Data, pp. 271-282, 1998.

[68] Y. Zhao, K. Ramasamy, K. Tufte, and J.F. Naughton, “Array-Based Evaluation of Multidimensional Queries in Object Relational Database Systems," Proc. Int'l Conf. Data Eng., 1998.

[69] Y. Zhuge and H. Garcia-Molina, "Graph Structural Views and Their Incremental Maintenance," Proc. Int'l Conf. Data Eng., 1998.

[70] Y. Zhuge, J.L. Wiener, and H. Garcia-Molina, "Multiple View Consistence for Data Warehousing," Proc. Int'l Conf. Data Eng., 1997.

[71] H. Zimmerman, Fuzzy Set Theory and Its Applications. Kluwer, Norwell Press, Inc., 1985.

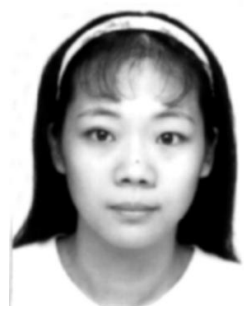

Ling Feng received the $\mathrm{BSc}$ and $\mathrm{PhD}$ degrees in computer science from Huazhong University of Science and Technology, China, in 1990 and 1995. She is currently an associate professor of computer science at the University of Twente in the Netherlands. Her research interests include data warehouses, data mining and its applications, distributed object-oriented database management systems, knowledge-based information systems, and Web-based information technologies. She has been engaged in 14 different international and national projects as a principal investigator, project leader, chief designer and developer, and has published more than 60 research papers in international and national conferences and journals. She is a member of the ACM, IEEE and AIS.

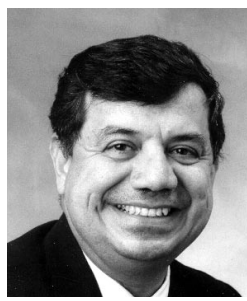

Tharam S. Dillon is the professor of Computing at Hong Kong Polytechnic University and the professor of Computer Science and Computer Engineering at La Trobe University in Melbourne, Australia, and the director of the Applied Computing Research Institute. His research interests include data mining, internet computing, e-commerce, hybrid neuro-symbolic systems, neural nets, software engineering, and database systems and computer networks. He has also worked with industry and commerce in developing systems in telecommunications, health care systems, e-commerce, logistics, power systems, and banking and finance. He is editor-in-chief of the International Journal of Computer Systems Science and Engineering and the International Journal of Engineering Intelligent Systems, as well as coeditor of the Journal of Electric Power and Energy Systems. He is an advisory editor of the IEEE Transactions on Neural Networks in the US. $\mathrm{He}$ is on the advisory editorial board of Applied Intelligence published by Kluwer in the US and Computer Communications published by Elservier in the UK. He has published more than 400 papers in international and national journals and conferences and has written four books and edited five other books. He is a fellow of the IEEE, the Institution of Engineers (Australia), and the Australian Computer Society.

$\triangleright$ For more information on this or any computing topic, please visit our Digital Library at http://computer.org/publications/dilb. 